\title{
Electromagnetic form factors of the $\Omega^{-}$baryon in the spacelike and timelike regions
}

\author{
G. Ramalho(1) \\ Laboratório de Física Teórica e Computacional-LFTC, Universidade Cruzeiro do Sul \\ and Universidade Cidade de São Paulo, 01506-000 São Paulo, SP, Brazil
}

(Received 9 January 2021; accepted 3 March 2021; published 23 April 2021)

\begin{abstract}
We present complete calculations of the electromagnetic form factors of the $\Omega^{-}$in the spacelike region and in the timelike region. The four elastic form factors: electric charge $\left(G_{E 0}\right)$, magnetic dipole $\left(G_{M 1}\right)$, electric quadrupole $\left(G_{E 2}\right)$, and magnetic octupole $\left(G_{M 3}\right)$, are estimated within the covariant spectator quark model, in terms of the square momentum transfer $q^{2}$. The free parameters of the $\Omega^{-}$wave function, including a $S$-wave state and two independent $D$-wave states radial wave functions and the admixture coefficients, are fixed by the comparison with the lattice QCD data in the spacelike region $\left(Q^{2}=-q^{2} \leq 0\right)$ and with the recent $e^{+} e^{-} \rightarrow \Omega^{-} \bar{\Omega}^{+}$data from CLEO in the timelike region $\left(q^{2}>0\right)$. The estimates in the timelike region for square momentum transfer $q^{2} \geq 4 M_{\Omega}^{2}$ are based on large- $q^{2}$ asymptotic relations $\left(M_{\Omega}\right.$ is the $\Omega^{-}$mass). We examine also the impact of the large- $Q^{2}$ correlations between different form factors and analyze the possible solutions. The electric quadrupole and the magnetic octupole moments of the $\Omega^{-}$and the $e^{+} e^{-} \rightarrow \Omega^{-} \bar{\Omega}^{+}$integrated cross sections for very large $q^{2}$ are estimated based on the model results.
\end{abstract}

DOI: 10.1103/PhysRevD.103.074018

\section{INTRODUCTION}

The study of the electromagnetic structure of the $\Omega^{-}$, composed by three valence strange quarks, is very challenging. Although the $\Omega^{-}$is the more stable known baryon with spin $3 / 2$ [longer mean life than the $\Delta(1232)$ ], its physical properties are almost unknown, apart the charge and the magnetic moment [1-11]. [The mean life of the $\Omega^{-}$is $8 \times 10^{-11} \mathrm{~s}$, and the mean life of the $\Delta(1232)$ is $6 \times 10^{-24} \mathrm{~s}$. The first measurement of the $\Omega^{-}$effective form factor $\left|G\left(q^{2}\right)\right|$ in the timelike region at CLOE ( $e^{+} e^{-} \rightarrow \Omega^{-} \bar{\Omega}^{+}$reactions) [12] opens a new window to probe the internal structure of the $\Omega^{-}$and the properties of the form factors at large $\left|q^{2}\right|$. The effective form factor $\left|G\left(q^{2}\right)\right|$ is determined by a combination of the four $\Omega^{-}$ electromagnetic form factors.

Additional information about the $\Omega^{-}$electromagnetic structure can be obtained from lattice QCD simulations, which can today be performed at the physical baryon mass in the spacelike region $\left(Q^{2} \geq 0\right)[7,13]$. These simulations can be regarded as a good representation of the physical baryon because they are performed at the physical strange quark mass and also because the effects of the meson cloud

Published by the American Physical Society under the terms of the Creative Commons Attribution 4.0 International license. Further distribution of this work must maintain attribution to the author(s) and the published article's title, journal citation, and DOI. Funded by SCOAP ${ }^{3}$. excitation of the baryon core are expected to be small (heavy meson excitations are suppressed according with chiral perturbation theory) $[14,15]$.

The measurement of the $\Omega^{-}$electromagnetic form factors for finite $Q^{2}=-q^{2}$ is a hard task due to the difficulty in creating strange baryon targets which may be scattered by electron beams $[11,14,16]$. The long life of the $\Omega^{-}$can, however, be used to obtain accurate determinations of the magnetic moment [8-10].

A question that can be raised is how well we can estimate today the $\Omega^{-}$electromagnetic form factors $G_{E 0}, G_{M 1}, G_{E 2}$, and $G_{M 3}$ [7,17-22] in the different kinematic regions. In the present work, we combine the present knowledge on the $\Omega^{-}$, including lattice QCD simulations in the spacelike region, measurements of the $\Omega^{-}$effective form factor in the timelike region, and the expected analytic behavior for very large $\left|Q^{2}\right|$, to shed some light on the dependence of the electric quadrupole and the magnetic octupole form factors on $Q^{2}$. The constraints associated with the electromagnetic form factors at the large $\left|Q^{2}\right|$ prove to have an important role in the shape of the form factors. The timelike data are very pertinent to the process because they contain information about the large- $\left|Q^{2}\right|$ region and provide a unique test to the shape of the form factors in an extreme regime. Although lattice QCD simulations can be used to infer the dependence of the form factors on $Q^{2}$, they are presently limited in precision above $Q^{2}=2 \mathrm{GeV}^{2}$ [7].

The magnetic moment of the $\Omega^{-}$, the electric charge $\left(G_{E 0}\right)$, and the magnetic dipole $\left(G_{M 1}\right)$ form factors have been estimated using several frameworks [1,4,23-43]. 
Although there are a few estimates of the $\Omega^{-}$quadrupole form factors [44-55], including extrapolations from lattice QCD simulations [5,7,13], and of the $\Omega^{-}$octupole moment [52,56-58], the information about the functions $G_{E 2}$ and $G_{M 3}$ is scarce. Accurate lattice QCD simulations are at the moment limited to $0 \leq Q^{2} \leq 2 \mathrm{GeV}^{2}$. The only available lattice QCD simulation for $G_{M 3}$, gives $G_{M 3}\left(0.23 \mathrm{GeV}^{2}\right)=$ $1.25 \pm 7.50$ [13]. There is then all the interest in studying the function $G_{M 3}$, including the region near $Q^{2}=0$ and the respective falloff with $Q^{2}$. Experiments in facilities like $B A B A R$ [59], BES III [60], CLEO [12,61], and PANDA [62] based on $e^{+} e^{-}$collisions can also be used to access the electromagnetic structure of the $\Omega^{-}[6,61]$.

The $e^{+} e^{-} \rightarrow B \bar{B}$ experiments, where $B$ is a generic baryon, opens a new window to probe the electromagnetic structure of hyperons [12,63], hardly accessed in the spacelike region [11]. Of particular interest is the opportunity to study the correlations between different valence quark compositions, including quark pairs and others $[12,61,64-68]$. The first theoretical estimates of the $e^{+} e^{-} \rightarrow B \bar{B}$ cross sections and hyperon effective form factor, in the timelike region, were based on vector meson dominance (VMD) models [69,70]. More recently, with the emergence of accurate data for a variety of hyperons, new models have been proposed [6,71-74], including improved VMD models [75-80]. Most of these studies focus on the $\Lambda$ and $\Sigma$ systems. Theoretical studies of the $\Omega^{-}$electromagnetic properties in the timelike region are rare $[6,12,61,72]$.

Our calculations of the $\Omega^{-}$electromagnetic form factors follow the formalism of the covariant spectator quark model [15,81-83] for spin-3/2 baryons. In the formalism, the $\Omega^{-}$wave function is represented by a combination of a dominate $S$-wave state and two $D$-wave states $[4,5,21,84]$. The mixture parameters and the radial structure of the three components are determined by fits to the available data (the magnetic moment, lattice QCD, and effective timelike form factor) as well by the expected behavior of the form factors for very large $Q^{2}$. At large $Q^{2}$, we consider also a relation between the form factors $G_{M 1}$ and $G_{M 3}$, derived from the asymptotic behavior of the helicity transition amplitudes at large $Q^{2}$. At the end, we use our best parametrization to make predictions to the electric quadrupole and magnetic octupole moments of the $\Omega^{-}$and the effective form factor $\left|G\left(q^{2}\right)\right|$ at large $q^{2}$.

Although the present analysis is dominated by spacelike data, we conclude that the information about the function $G_{M 3}$ and the effective form factor $\left|G\left(q^{2}\right)\right|$ is important to determine the shape of the form factors. The timelike and $G_{M 3}$ data are represented by three points, while the remaining data (lattice QCD) are represented by 100 points. We conclude also that accurate lattice QCD calculations of $G_{M 3}$, possible with the present state-of-the-art methods, and measurements of the $e^{+} e^{-} \rightarrow \Omega^{-} \bar{\Omega}^{+}$cross sections at large $q^{2}$ can further help to infer the shape of the form factors at large $\left|Q^{2}\right|$ and to reduce the uncertainty of $G_{M 3}(0)$.
The present article is organized as follows. In the next section, we discuss in detail the available experimental and theoretical information about the $\Omega^{-}$form factors. In Sec. III, we discuss the covariant spectator quark model and the formalism associated to baryons with spin $3 / 2$ and positive parity. The calculations of the $\Omega^{-}$electromagnetic form factors in the spacelike region $\left(Q^{2} \geq 0\right)$ are presented in Sec. IV. The extension of the model for the timelike region $\left(q^{2}=-Q^{2}>0\right)$ and our final results are presented and discussed in Sec. V. In Sec. VI, we present the outlook and conclusions.

\section{ELECTROMAGNETIC STRUCTURE OF THE $\Omega^{-}$BARYON}

The $\Omega^{-}$is a baryon with spin $3 / 2$ and positive parity $\left(J^{P}=\frac{3}{2}^{+}\right)$. As a consequence, the transition current is characterized by four independent structure functions dependent on $Q^{2}$ [7,17-19]. The most common representation of those structure functions is the multipole form factor representation, where the $\Omega^{-}$structure is described by the electric charge $\left(G_{E 0}\right)$, magnetic dipole $\left(G_{M 1}\right)$, electric quadrupole $\left(G_{E 2}\right)$, and magnetic octupole $\left(G_{M 3}\right)$ form factors $[5,7,20,21]$. The definition of the multipole form factors is presented in Appendix A. The electric charge and the magnetic dipole form factors provide information about the distribution of charge and magnetism inside the baryons. The electric quadrupole and magnetic octupole measure the deviations from the distributions from a symmetrical form $\left(G_{E 2} \neq 0\right.$ and $\left.G_{M 3} \neq 0\right)$, providing a direct evidence of the deformation of the baryons $[7,19,22,49,56,85]$.

As pointed out already, except for the electron-positron collisions [6,12], the $\Omega^{-}$baryon is difficult to produce in the laboratory $[8,9,11]$, due the structure based on three strange quarks. For this reason, the electromagnetic structure of the $\Omega^{-}$is almost unknown, except for the charge $(-e)$ and the magnetic moment $\mu_{\Omega}$.

We review next our sources of information about the $\Omega^{-}$ electromagnetic structure.

\section{A. Experimental data}

The long lifetime $\left(\tau_{\Omega} \simeq 8 \times 10^{-11} \mathrm{~s}\right.$, decay by weak interaction) [10] allows a precise determination of the $\Omega^{-}$magnetic moment. The $\Omega^{-}$magnetic moment has been measured a few times with different precisions $[8,9]$. The Particle Data Group (PDG) presents the world's average, $\mu_{\Omega}=(-2.02 \pm 0.05) \mu_{N}$, in nucleon magnetons $\left(\mu_{N} \equiv \frac{e}{2 M_{N}}\right.$, where $M_{N}$ is the nucleon mass and $e$ is the elementary charge). In the present work, we use the result from PDG [10] corresponding to

$$
G_{M 1}(0)=-3.60 \pm 0.09,
$$

based on $\mu_{\Omega} \equiv G_{M 1}(0) \frac{e}{2 M_{\Omega}}$. 


\section{B. Results from lattice QCD simulations}

Since the structure of the $\Omega^{-}$is dominated by three strange valence quarks, one assumes that the electromagnetic form factors can in a good approximation be simulated by lattice QCD calculations at the strange quark physical mass. These simulations are possible in the present days, as shown in several lattice QCD simulations $[7,86]$. Although it may be argued that the valence quark structure is not the complete picture and that sea quark effects must also be taken into account, it is known that those effects are dominated by the kaon cloud, since the pions cannot be produced directly by three strange quark cores. The kaon and eta cloud effects, however, are suppressed according to chiral perturbation theory [87-89]. The conclusion is then that the lattice QCD simulations at the physical strange quark mass can be interpreted as an accurate simulation of the physical results and that no extrapolation of the results is necessary The only limitation of these calculations is the intrinsic errors associated with lattice QCD simulations, such as the size of the lattice spacing and the finite volume of the simulations.

In this work, we consider the more consistent simulation of the $\Omega^{-}$electromagnetic form factors from Alexandrou et al. [7], as a reliable representation of the $\Omega^{-}$physical electromagnetic form factors. Early lattice QCD simulations can be found in Refs. [23,48].

The simulations from Ref. [7] are based on two unquenched methods: the domain-wall fermions (DWF) method and the hybrid action method. The simulations from Ref. [7] are restricted to the form factors $G_{E 0}, G_{M 1}$, and $G_{E 2}$. These simulations provide data for $Q^{2}$ up to $4 \mathrm{GeV}^{2}$, but only the data for $Q^{2} \leq 2 \mathrm{GeV}^{2}$ are relatively precise. The hybrid action simulations correspond to $m_{\pi}=0.353 \mathrm{MeV}$. For the DWF, there are simulations for $m_{\pi}=297,330$, and $355 \mathrm{MeV}$. Ideally, we should select the simulations corresponding to the lower pion mass for each method. Unfortunately, some datasets do not include results for all form factors, or the data statistic are poor. To obtain the complete picture of the form factors $G_{E 0}, G_{M 1}$, and $G_{E 2}$, in this work, we use then the four sets of lattice QCD data from Ref. [7].

The only direct information about the octupole magnetic form factors come from lattice QCD simulations from Boinepalli et al. [13] for the $\Delta^{-}$form factors in the $S U(3)$ limit, when the pion mass is $m_{\pi}=0.697 \mathrm{GeV}$. In this point, the $d$ quarks and $s$ quarks have the same properties and masses, and the $\Delta^{-}$ structure resembles the $\Omega^{-}$structure. The simulation from Ref. [13] overestimates the physical $\Omega^{-}$mass. It provides, nevertheless, the only available estimate of the form factor $G_{M 3}$ based on QCD first principles. These estimates are performed at one single point $Q^{2}=$ $0.23 \mathrm{GeV}^{2}$. The result of the magnetic octupole form factor is $G_{M 3}\left(Q^{2}\right)=1.25 \pm 7.50$ [13].

\section{C. $\Omega^{-}$data in the timelike region}

In recent years, there have been important experimental developments in the study of the baryon structure in the timelike region, based on the electron-positron collisions in facilities like $B A B A R$, BES III, and CLEO [12,59-61,90]. From the $e^{+} e^{-} \rightarrow B \bar{B}$ reactions, one has access to the electromagnetic structure of the baryon $B$, in the region $Q^{2}=-q^{2}<0$. The threshold of the transition is $q^{2}=$ $-Q^{2} \geq 4 M_{B}^{2}$ ( $M_{B}$ is the baryon mass). Of particular interest has been the production of $\Omega^{-}$baryon and the respective antistate in CLEO [61].

In those experiments, the integrated $e^{+} e^{-} \rightarrow B \bar{B}$ cross section in the $e^{+} e^{-}$center-of-mass frame becomes $[6,61,90]$

$$
\sigma\left(q^{2}\right)=\frac{4 \pi \alpha^{2} \beta C}{3 q^{2}}\left(1+\frac{1}{2 \tau_{T}}\right)\left|G\left(q^{2}\right)\right|^{2},
$$

where $G\left(q^{2}\right)$ is an effective form factor, $\tau_{T}=\frac{q^{2}}{4 M_{B}^{2}}, \alpha \simeq \frac{1}{137}$ is the fine-structure constant, $\beta$ is a kinematic factor defined by $\beta=\sqrt{1-\frac{1}{\tau_{T}}}$, and $C$ is a factor which depends on the charge of $B[6,90]$. For large $q^{2}$, one has $C \simeq 1$.

The effective form factor $G\left(q^{2}\right)$ for baryons with spin $1 / 2$ and positive parity $\left(J^{P}=\frac{1}{2}^{+}\right.$states $)$takes the form $[12,59,91-93]$

$$
\begin{aligned}
\left|G\left(q^{2}\right)\right|^{2} & =\left(1+\frac{1}{2 \tau_{T}}\right)^{-1}\left[\left|G_{M}\left(q^{2}\right)\right|^{2}+\frac{1}{2 \tau_{T}}\left|G_{E}\left(q^{2}\right)\right|^{2}\right], \\
& =\frac{2 \tau_{T}\left|G_{M}\left(q^{2}\right)\right|^{2}+\left|G_{E}\left(q^{2}\right)\right|^{2}}{2 \tau_{T}+1}
\end{aligned}
$$

where $G_{E}$ and $G_{M}$ are the electric charge and magnetic dipole form factors.

Equation (2.3) is still valid for $\frac{3}{2}^{+}$states, if we use the replacements [69]

$$
\begin{gathered}
\left|G_{E}\right|^{2} \rightarrow 2\left|G_{E 0}\right|^{2}+\frac{8}{9}\left(\tau_{T}\right)^{2}\left|G_{E 2}\right|^{2}, \\
\left|G_{M}\right|^{2} \rightarrow \frac{10}{9}\left|G_{M 1}\right|^{2}+\frac{32}{5}\left(\tau_{T}\right)^{2}\left|G_{M 3}\right|^{2} .
\end{gathered}
$$

\section{Perturbative QCD constraints}

Additional information about the $\Omega^{-}$form factors come from perturbative QCD (pQCD) for very large $Q^{2}$ (or $q^{2}$ ) [94-96]. As for the case of the nucleon, where pQCD estimates show that $G_{E}, G_{M} \propto 1 / Q^{4}$, also in the case of $\frac{3}{2}^{+}$baryons, one can estimate the falloff of the form factors for very large $Q^{2}$. For the $\frac{3}{2}{ }^{+}$resonances, one obtains 


$$
\begin{aligned}
& G_{E 0}, G_{M 1} \propto \frac{1}{Q^{4}}, \\
& G_{E 2}, G_{M 3} \propto \frac{1}{Q^{6}},
\end{aligned}
$$

apart logarithmic corrections [94,96]. [Meaning that the leading-order dependence can include factors $\log \left(Q^{2}\right)$, or powers of $\log \left(Q^{2}\right)$, which are negligible in comparison with $Q=\sqrt{Q^{2}}$ ]. The corollary of these results is that $G\left(q^{2}\right) \propto 1 / q^{4}$ for very large $q^{2}$, as a consequence of the asymptotic relations between spacelike and timelike form factors [6]. Those relations are discussed in Sec. V B.

The analysis of the helicity transition amplitudes at large $Q^{2}$ imposes, however, a constraint stronger than (2.6) and (2.7). From the study of the asymptotic behavior of the helicity transition amplitudes [94,95], one concludes that the magnetic-type form factors are related for very large $Q^{2}$ by

$$
G_{M 1}=\frac{4}{5} \tau G_{M 3},
$$

where $\tau=\frac{Q^{2}}{4 M^{2}}$. In this notation, $\tau=-\tau_{T}$. The previous relation is derived in Appendix A. The error expected in the relation is terms of the order of $1 / Q^{6}$.

The condition (2.8) may look surprising at first. One needs to keep in mind, however, that correlations between transition form factors at large $Q^{2}$ are common on electromagnetic transitions between baryon states. Examples are some $\gamma^{*} N \rightarrow N^{*}$ transitions, when $N^{*}$ are $\frac{3}{2}^{+}$and $\frac{3}{2}^{-}$states, as the $\Delta(1232)$ and the $N(1520)$. In those cases, one has $G_{M} \simeq-G_{E}$, for very large $Q^{2}$ [84,97-99]. Although those relations are related to the falloff of the transverse transition amplitudes $\left(A_{1 / 2}\right.$ and $\left.A_{3 / 2}\right)$ [100], those constraints are only taken into account implicitly in some quark models and in some parametrizations of the data [99]. The condition (2.8) is also valid for the $\Delta(1232)$ elastic form factors [20,21] and for the other decuplet baryon members.

As far as we know, the constraint (2.8) has not been discussed in the literature, but it has a significant impact on our final results for the $\Omega^{-}$electromagnetic form factors. The relation (2.8) is, however, the consequence of the natural order of the transition amplitudes between $\frac{3}{2}+$ and $\frac{3}{2}+$ baryon states (see Appendix A).

\section{COVARIANT SPECTATOR QUARK MODEL}

In the present section, we discuss the formalism associated with the covariant spectator quark model $[4,15,81]$. The model was developed within the covariant spectator theory [101]. In the framework, the baryons are interpreted as systems of three-constituent quarks where a quark is free to interact with electromagnetic probes in relativistic impulse approximation [4,81,83]. Integrating over the degrees of freedom of the noninteracting quarks, one reduces the three-quark system to a quark-diquark system where the spectator quark pair is represented by an onmass-shell diquark with an average mass $m_{D}[4,81,82]$. One obtains then an effective quark-diquark wave function, free of singularities which describe the quark confinement implicitly [81,82].

The wave functions of the baryons are built according to the spin-flavor-radial symmetries where the radial wave functions are determined phenomenologically by the experimental data or by lattice QCD data for some ground state systems $[4,14,15,84,102,103]$. In the electromagnetic interaction with the quarks, we take into account the structure related to the gluon and quark-antiquark dressing. To parametrize this structure, we use a form based on VMD to represent the constituent quark electromagnetic form factors $[4,81,103]$.

The formalism has been applied extensively to the study of the electromagnetic structure of several baryons in the spacelike region $\left(Q^{2} \leq 0\right)$ [5,81-84,98,103-109] and in the timelike region $\left(Q^{2}<0\right)$ [6,110-112]. The formalism has also been used in the study of the spacelike electromagnetic form factors of baryons in the lattice QCD regime $[4,102,103,106]$ and in the nuclear medium [113].

\section{A. Transition current}

In the relativistic impulse approximation, the transition current between two baryon states, $B$ and $B^{\prime}$, described by quark-diquark wave functions, $\Psi_{B^{\prime}}$ and $\Psi_{B}$, takes the form $[4,81,82]$

$$
J_{B^{\prime}, B}^{\mu}=3 \sum_{\Gamma} \int_{k} \bar{\Psi}_{B^{\prime}}\left(P_{+}, k\right) j_{q}^{\mu} \Psi_{B}\left(P_{-}, k\right),
$$

where $P_{+}, P_{-}$, and $k$ are the final, initial, and diquark momenta; $j_{q}^{\mu}$ is the quark current operator; and $\Gamma$ labels the diquark scalar and vector components. The factor 3 takes into account the contributions associated with the different diquark pairs. The integral symbol represents the covariant integration on the on-shell diquark momentum.

When we include the explicit form of the wave functions $\Psi_{B^{\prime}}$ and $\Psi_{B}$, we reduce $J_{B^{\prime}, B}^{\mu}$ to a Lorentz-invariant form projected into the asymptotic states of $B^{\prime}$ and $B$. Each gauge-invariant term defines an independent form factor. For details about the $\frac{3}{2}+$ elastic form factors, check Refs. [17-21] and Appendix A.

In the following, we consider the elastic case $\left(B^{\prime}=B\right)$, since our focus is the electromagnetic form factors of $\frac{3}{2}+$ baryons.

The quark current operator has the generic form

$$
j_{q}^{\mu}=j_{1}\left(Q^{2}\right) \gamma^{\mu}+j_{2}\left(Q^{2}\right) \frac{i \sigma^{\mu \nu} q_{\nu}}{2 M_{N}},
$$

where $M_{N}$ is the nucleon mass, as before, and $j_{1}$ and $j_{2}$ are the Dirac and Pauli $S U(3)$ flavor operators, respectively. 
Equation (3.2) was defined for the first time for the study of the nucleon elastic form factors [81]. The quark current $j_{q}^{\mu}$ was later extended to baryons with strange quarks $[4,6,103,113]$.

The operators $j_{i}(i=1,2)$ can be decomposed as

$j_{i}\left(Q^{2}\right)=\frac{1}{6} f_{i+}\left(Q^{2}\right) \lambda_{0}+\frac{1}{2} f_{i-}\left(Q^{2}\right) \lambda_{3}+\frac{1}{2} f_{i 0}\left(Q^{2}\right) \lambda_{s}$,

where

$$
\begin{array}{ll}
\lambda_{0}=\left(\begin{array}{lll}
1 & 0 & 0 \\
0 & 1 & 0 \\
0 & 0 & 0
\end{array}\right), & \lambda_{3}=\left(\begin{array}{ccc}
1 & 0 & 0 \\
0 & -1 & 0 \\
0 & 0 & 0
\end{array}\right), \\
\lambda_{s}=\left(\begin{array}{ccc}
0 & 0 & 0 \\
0 & 0 & 0 \\
0 & 0 & -2
\end{array}\right), &
\end{array}
$$

are the flavor operators acting on the quark wave function in the flavor space, $q=(u d s)^{T}$. The functions $f_{i+}, f_{i-}$ $(i=1,2)$ represent the quark isoscalar and isovector form factors, respectively, based on the combinations of the quarks $u$ and $d$ [81]. The functions $f_{i 0}(i=1,2)$ represent the structure associated with the strange quark [4].

The quark isoscalar and isovector (light) form factors are important for the study of the nucleon, the octet baryon, the decuplet baryon, and the transitions between the octet baryon and decuplet baryon $[4,81,103,105,113]$ but are not relevant to the present work.

When we consider a baryon composed exclusively of strange quarks, like the $\Omega^{-}$baryon, only the terms in $f_{i 0}$ survive when we project $j_{i}$ into the flavor wave functions.

To parametrize the strange the strange quark form factors, we use the form inspired by the VMD mechanism [4]

$$
\begin{gathered}
f_{10}=\lambda_{q}+\left(1-\lambda_{q}\right) \frac{m_{\phi}^{2}}{m_{\phi}^{2}+Q^{2}}+c_{0} \frac{M_{h}^{2} Q^{2}}{\left(M_{h}^{2}+Q^{2}\right)^{2}}, \\
f_{20}=\kappa_{s}\left\{d_{0} \frac{m_{\phi}^{2}}{m_{\phi}^{2}+Q^{2}}+\left(1-d_{0}\right) \frac{M_{h}^{2}}{M_{h}^{2}+Q^{2}}\right\},
\end{gathered}
$$

where $m_{\phi}$ and $M_{h}$ are the vector meson masses, corresponding, respectively, to the light vector meson ( $\phi$ meson, associated with an $s \bar{s}$ state) and an effective heavy meson with mass $M_{h}=2 M_{N}$, which simulate the short-range phenomenology. The parameter $\lambda_{q}$ is determined by the study of deep inelastic scattering [81]; $\kappa_{s}, c_{0}$, and $d_{0}$ are determined by the study of the decuplet baryon electromagnetic form factors [4], based on the lattice QCD simulations from Ref. [13]. The calibration of the strange quark form factors takes into account also the experimental value for the $\Omega^{-}$magnetic moment [4]. The numerical values of the free parameters are $\lambda_{q}=1.21, \kappa_{s}=1.462$, $c_{0}=4.427$, and $d_{0}=-1.860$.

\section{B. Wave functions of spin- $-\frac{3}{2}+$ baryons}

We now review the formalism associated with the $\frac{3}{2}+$ baryon states, developed in previous works in the study of the $\Delta(1232)$ and the $\Omega^{-}$systems $[4,5,20,21,83,84,102]$. We assume that the state corresponds to the $\frac{3}{2}+$ baryon ground state (no radial excitations). The differences to the previous works are in the flavor states $\left(\Omega^{-}\right.$system) and in the radial wave functions.

We can decompose the wave functions of the $\frac{3}{2}^{+}$baryon $B$ into three main components, associated with a mixture of an $S$ state and two $D$ states, labeled here as $D 3$ and $D 1$ states, for the quark-diquark relative motion [5]

$$
\Psi_{B}(P, k)=N\left[\Psi_{S}(P, k)+a \Psi_{D 3}(P, k)+b \Psi_{D 1}(P, k)\right],
$$

where $a$ and $b$ are the $D$-state mixture coefficients of the states $D 3$ and $D 1$, respectively, and $N$ is the normalization constant (assuming that the individual states are properly normalized). The state $D 3$ describes the configuration where the sum of the spin of the three quarks is $3 / 2$. The state $D 1$ describes the configuration where the sum of the spin of the three quarks is $1 / 2$.

The interpretation of the states as $S$ - and $D$-wave components comes from the structure of the states in the rest frame. In a moving frame, the intrinsic $S$ - and $D$-wave states are modified, and other partial waves are generated $[83,84]$.

In the following, we refer states of core-spin $\mathcal{S}$ to refer to states where the sum of the spin of the three quarks is $\mathcal{S}$ (ignoring the relative angular momentum). The possible states for systems of three quarks are then $\mathcal{S}=1 / 2$ or $\mathcal{S}=3 / 2$.

In the present study, we are not taking into account contributions associated with $P$-wave states. Those contributions may be relevant for the nucleon and the octet baryon $[82,114]$ but appear to not be so relevant for the $\frac{3}{2}+$ states. Notice that the $\gamma^{*} N \rightarrow \Delta(1232)$ transition can be described accurately by a combination of $S$ and $D$ states on the $\Delta(1232)$ wave function $[84,102,104]$.

The explicit expressions for the $S, D 3$, and $D 1$ components of the wave functions are presented next.

\section{S-state wave function}

The $S$-state contribution to the wave function of the baryon $B$, corresponding to a quark-diquark system with relative angular momentum $L=0$, can be written as $[4,83,84]$

$$
\Psi_{S}(P, k)=-\psi_{S}(P, k)|B\rangle_{F} \varepsilon_{\lambda P}^{\alpha *} u_{\alpha}(P, s),
$$


where $\psi_{S}(P, k)$ is the $S$-state radial wave function, $|B\rangle_{F}$ is the baryon $B$ flavor wave function, $\varepsilon_{\lambda P}^{\alpha *}$ is the diquark polarization state $(\lambda=0, \pm)$ in the fixed-axis basis $[81,115]$, $u_{\alpha}(P, s)$ is the Rarita-Schwinger spinor [116,117], and $s$ is the spin projection of the baryon. The indices $\lambda$ and $s$ are omitted on $\Psi_{S}(P, k)$ for simplicity.

Equation (3.8) generalizes the nonrelativistic structure of a three-quark wave function of the $\frac{3}{2}+$ ground-state baryon and satisfies the Dirac equation $\left(\not P-M_{B}\right) \Psi_{S}(P, k)=0$ $[83,84,115]$.

\section{D-state wave functions}

The construction of the states associated to quarkdiquark configurations with a relative angular momentum $L=2$ requires the derivation of a $\mathcal{D}$-state operator and also a consideration of projectors $\mathcal{P}_{1 / 2}$ and $\mathcal{P}_{3 / 2}$, which decompose generic states into their components into states of core-spin $1 / 2$ and states of core-spin $3 / 2$, respectively.

The $\mathcal{D}$-state operator can be expressed in terms of the momentum [84]

$$
\tilde{k}^{\alpha}=k^{\alpha}-\frac{P \cdot k}{M_{B}^{2}} P^{\alpha}
$$

which can be used for the initial diquark $\left(P=P_{-}\right.$and $\left.\tilde{k} \rightarrow \tilde{k}_{-}\right)$or the final diquark $\left(P=P_{+}\right.$and $\left.\tilde{k} \rightarrow \tilde{k}_{+}\right)$. At the baryon rest frame, $\tilde{k}=(0, \mathbf{k})$. Using this notation, we can define the $\mathcal{D}$-state operator [84] as

$$
\mathcal{D}^{\alpha \beta}(P, k)=\tilde{k}^{\alpha} \tilde{k}^{\beta}-\frac{1}{3} \tilde{k}^{2} \tilde{g}^{\alpha \beta},
$$

where

$$
\tilde{g}^{\alpha \beta}=g^{\alpha \beta}-\frac{P^{\alpha} P^{\beta}}{M_{B}^{2}} .
$$

Note that Eqs. (3.10) and (3.11) can be defined in the initial state $\left(P_{-}, \tilde{k}_{-}\right)$or in the final state $\left(P_{+}, \tilde{k}_{+}\right)$.

To separate the states of core-spin $1 / 2$ from the states of core-spin $3 / 2$, we consider the two projectors,

$$
\begin{gathered}
\left(\mathcal{P}_{3 / 2}\right)^{\alpha \beta}=\tilde{g}^{\alpha \beta}-\frac{1}{3} \tilde{\gamma}^{\alpha} \tilde{\gamma}^{\beta}, \\
\left(\mathcal{P}_{1 / 2}\right)^{\alpha \beta}=\frac{1}{3} \tilde{\gamma}^{\alpha} \tilde{\gamma}^{\beta},
\end{gathered}
$$

where

$$
\tilde{\gamma}^{\alpha}=\gamma^{\alpha}-\frac{\not P P^{\alpha}}{M_{B}^{2}}
$$

The properties of these projectors are known in the literature $[83,84,117]$.
To represent the two $D$ states in a compact form, it is convenient to define also the state

$$
W^{\alpha}(P, k ; s)=\mathcal{D}^{\alpha \beta}(P, k) u_{\beta}(P, s) .
$$

One obtains two different states, $D 3$ and $D 1$, when we use the core-spin projectors $\mathcal{P}_{3 / 2}$ and $\mathcal{P}_{1 / 2}$, defined by Eqs. (3.12) and (3.13):

$$
\begin{aligned}
& W_{D 3}^{\alpha}(P, k ; s)=\left(\mathcal{P}_{3 / 2}\right)^{\alpha \beta} W_{\beta}(P, k ; s), \\
& W_{D 1}^{\alpha}(P, k ; s)=\left(\mathcal{P}_{1 / 2}\right)^{\alpha \beta} W_{\beta}(P, k ; s) .
\end{aligned}
$$

The wave functions of the states $D 3$ and $D 1$ can now be written as $[21,84]$

$\Psi_{D 3}(P, k)=-3 \psi_{D 3}(P, k)|B\rangle_{F}\left(\varepsilon_{\lambda P}^{*}\right)_{\alpha} W_{D 3}^{\alpha}(P, k ; s)$,

$\Psi_{D 1}(P, k)=-3 \psi_{D 1}(P, k)|B\rangle_{F}\left(\varepsilon_{\lambda P}^{*}\right)_{\alpha} W_{D 1}^{\alpha}(P, k ; s)$,

where $\psi_{D 3}$ and $\psi_{D 1}$ are the $D 3$ and $D 1$ radial wave functions, respectively, and the factor -3 was included by convenience in order to mimic the form of $\Psi_{S}$ from (3.8) and to simplify the normalization condition of the two $D$ states [84]. As for the $S$ state, the wave functions $\Psi_{D 3}(P, k)$ and $\Psi_{D 1}(P, k)$ are both solutions of the Dirac equation.

The normalization of the radial wave functions is discussed in the next section [see Eqs. (4.5)], along with the discussion of the form of the radial wave functions.

One can demonstrate that the states $u^{\alpha}(P, s)$, $W_{D 3}^{\alpha}(P, k, s)$ are states with core-spin $3 / 2$, since the projection with $\mathcal{P}_{1 / 2}$ is zero, and the states are unchanged by the projector $\mathcal{P}_{3 / 2}$ [84]. As for the state $W_{D 1}^{\alpha}(P, k, s)$, it is a state with core-spin $1 / 2$, since the projection with $\mathcal{P}_{3 / 2}$ is zero, and it remains unchanged when projected by $\mathcal{P}_{1 / 2}$ [84]. Furthermore, it was proved that Eqs. (3.18) and (3.19) generalize the nonrelativistic wave function of threequark $D$ states ground state with core-spin $3 / 2$ and $1 / 2$, respectively [84].

\section{SPACELIKE MODEL FOR THE $\Omega^{-}$BARYON}

We discuss now the results of the covariant spectator quark model for the $\Omega^{-}$electromagnetic form factors in the spacelike region. We consider the $\Omega^{-}$wave function described by a combination of an $S$, a $D 3$, and a $D 1$ state for a $\frac{3}{2}^{+}$ground-state baryon, as in Eq. (3.7) with the flavor state $\left|B>_{F}=\right|$ sss $>$. In the following, we replace $B$ by $\Omega$ in the wave functions and masses.

The explicit expressions for the $\Omega^{-}$electromagnetic form factors are derived in Refs. [5,84]. The final results depend on the parametrization of the strange quark form factors (3.5) and (3.6) and on the form of the radial wave functions $\psi_{S}, \psi_{D 3}$, and $\psi_{D 1}$. 
Before presenting the final expressions to the electromagnetic form factors, we discuss the parametrizations to the radial wave functions.

\section{A. Radial wave functions}

Following the formalism of the covariant spectator quark model, we express the radial wave functions in terms of the dimensionless variable

$$
\chi=\frac{\left(M_{\Omega}-m_{D}\right)^{2}-(P-k)^{2}}{M_{\Omega} m_{D}} .
$$

This representation is justified in the cases that the baryons and the diquark are both on mass shell [15,81].

For the $S, D 3$, and $D 1$ states, we consider the radial wave functions [4],

$$
\begin{gathered}
\psi_{S}(P, k)=\frac{N_{S}}{m_{D}\left(\alpha_{1}+\chi\right)\left(\alpha_{2}+\chi\right)}, \\
\psi_{D 3}(P, k)=\frac{N_{D 3}}{m_{D}^{3}\left(\alpha_{3}+\chi\right)^{4}}, \\
\psi_{D 1}(P, k)=\frac{N_{D 1}}{m_{D}^{3}\left(\alpha_{4}+\chi\right)^{4}},
\end{gathered}
$$

where $N_{S}, N_{D 3}$, and $N_{D 1}$ are normalization constants and $\alpha_{i}$ $(i=1, \ldots, 4)$ are square momentum range parameters in units $M_{\Omega} m_{D}$. The factors $1 / m_{D}$ and $1 / m_{D}^{3}$ are included to ensure appropriate normalizations for the wave functions (dimensionless overlap integral functions).

The previous radial wave functions are normalized according with $[84,102]$

$$
\begin{aligned}
\int_{k}\left|\psi_{S}(\bar{P}, k)\right|^{2} & =1, \\
\int_{k} \tilde{k}^{4}\left|\psi_{D 3}(\bar{P}, k)\right|^{2} & =1, \\
\int_{k} \tilde{k}^{4}\left|\psi_{D 1}(\bar{P}, k)\right|^{2} & =1,
\end{aligned}
$$

where $\bar{P}$ represents the $\Omega^{-}$momentum at the rest frame: $\bar{P}=\left(M_{\Omega}, 0,0,0\right)$ and $\tilde{k}=(0, \mathbf{k})$.

The inspiration for Eq. (4.2) comes from the representation of the nucleon radial wave function, since it can be reduced to the Hulthen form in the nonrelativistic limit in the configuration space (difference of two Yukawa functions) [81]. Compared to our previous work on the $\Omega^{-}$ baryon with $D$ states [5], we kept the expression for the $D 3$ and $D 1$ radial wave functions but modified the form for $\psi_{S}$, which is now defined by the product of two different multipoles on the variable $\chi$.

The motivation to the new form is twofold: generate an asymptotic form for the $S$-state contribution compatible with the leading-order form factors at large $Q^{2}$ (proportional to $1 / Q^{4}$ ), and increase the flexibility of the fit, including two different momentum scale parameters. The parametrizations (4.2)-(4.4) are compatible with the expected falloffs (2.6) and (2.7) of the form factors for large $Q^{2}$, as discussed below. In the previous work [5], our main goal was the description of the $\Omega^{-}$in a limited region of $Q^{2}$.

The parametrizations (4.2)-(4.4) may, however, be incompatible with the relation (2.8). For that reason, we consider also an alternative parametrization of the $S$-state wave function and minor modifications in the $D$-state radial wave functions.

\section{B. Alternative parametrizations for the radial wave functions}

Alternative parametrizations for the $S, D 3$, and $D 1$ radial wave functions can be

$$
\begin{gathered}
\psi_{S}(P, k)=\frac{N_{S}}{m_{D}}\left[\frac{1}{\left(\alpha_{1}^{\prime}+\chi\right)\left(\alpha_{2}^{\prime}+\chi\right)^{2}}-\frac{r_{S}}{\left(\alpha_{1}+\chi\right)\left(\alpha_{2}+\chi\right)}\right], \\
\psi_{D 3}(P, k)=\frac{N_{D 3}}{m_{D}^{3}} \frac{1}{\left(\alpha_{1}+\chi\right)\left(\alpha_{2}+\chi\right)\left(\alpha_{3}+\chi\right)^{2}} \\
\psi_{D 1}(P, k)=\frac{N_{D 1}}{m_{D}^{3}} \frac{1}{\left(\alpha_{1}+\chi\right)\left(\alpha_{2}+\chi\right)\left(\alpha_{4}+\chi\right)^{2}}
\end{gathered}
$$

where $N_{S}, N_{D 3}$, and $N_{D 1}$ are new normalization constants; $\alpha_{1}^{\prime}$ and $\alpha_{2}^{\prime}$ are additional square momentum range parameters; and $r_{S}$ is a new adjustable parameter. The normalization constants are also determined by the conditions (4.5).

The parametrizations (4.6)-(4.8) are characterized by the common factors associated with the parameters $\alpha_{1}$ and $\alpha_{2}$. In Sec. V C, we show that in Eq. (4.6) the term associated with the parameters $\alpha_{1}$ and $\alpha_{2}$ dominates over the term with the associated with parameters $\alpha_{1}^{\prime}$ and $\alpha_{2}^{\prime}$, on the overlap integral, at large $Q^{2}$.

\section{C. $\boldsymbol{\Omega}^{-}$form factors}

The $\Omega^{-}$elastic form factors are calculated in a previous work [5] for a mixture of $S, D 3$, and $D 1$ states given by Eq. (3.7), in the first order of the coefficients $a$ and $b$. The approximation is justified for small admixture coefficients.

To represent the $\Omega^{-}$elastic form factors, it is convenient to define the functions $[5,20,21]$

$$
\tilde{e}_{\Omega}=-f_{10}\left(Q^{2}\right), \quad \tilde{\kappa}_{\Omega}=-\frac{M_{\Omega}}{M_{N}} f_{20}\left(Q^{2}\right)
$$

We use the tilde to represent functions of $Q^{2}$ without the explicit inclusion of the argument. Also useful for the representation of the electric-type and magnetic-type form factors are the combinations 


$$
\begin{gathered}
\tilde{g}_{\Omega}=\tilde{e}_{\Omega}-\tau \tilde{\kappa}_{\Omega}, \\
\tilde{f}_{\Omega}=\tilde{e}_{\Omega}+\tilde{\kappa}_{\Omega},
\end{gathered}
$$

where $\tau=\frac{Q^{2}}{4 M_{\Omega}^{2}}$. Note the similarity with the expressions for the electric and magnetic form factors of $\frac{1}{2}+$ baryons.

The covariant spectator quark model results for the $\Omega^{-}$ elastic form factors can now be written as [5]

$$
\begin{gathered}
G_{E 0}\left(Q^{2}\right)=N^{2} \tilde{g}_{\Omega} \mathcal{I}_{S}, \\
G_{M 1}\left(Q^{2}\right)=N^{2} \tilde{f}_{\Omega}\left[\mathcal{I}_{S}+\frac{4}{5} a \mathcal{I}_{D 3}-\frac{2}{5} b \mathcal{I}_{D 1}\right], \\
G_{E 2}\left(Q^{2}\right)=N^{2} \tilde{g}_{\Omega}(3 a) \frac{\mathcal{I}_{D 3}}{\tau}, \\
G_{M 3}\left(Q^{2}\right)=N^{2} \tilde{f}_{\Omega}\left[a \frac{\mathcal{I}_{D 3}}{\tau}+2 b \frac{\mathcal{I}_{D 1}}{\tau}\right],
\end{gathered}
$$

where $N^{2}$ is a normalization factor, and the overlap integral functions are determined by

$$
\begin{gathered}
\mathcal{I}_{S}=\int_{k} \psi_{S}\left(P_{+}, k\right) \psi_{S}\left(P_{-}, k\right), \\
\mathcal{I}_{D 3}=\int_{k} b\left(\tilde{k}_{+}, \tilde{q}_{+}\right) \psi_{D 3}\left(P_{+}, k\right) \psi_{S}\left(P_{-}, k\right), \\
\mathcal{I}_{D 1}=\int_{k} b\left(\tilde{k}_{+}, \tilde{q}_{+}\right) \psi_{D 1}\left(P_{+}, k\right) \psi_{S}\left(P_{-}, k\right) .
\end{gathered}
$$

The function $b\left(\tilde{k}_{+}, \tilde{q}_{+}\right)$has the form

$$
b\left(\tilde{k}_{+}, \tilde{q}_{+}\right)=\frac{3}{2} \frac{\left(\tilde{k}_{+} \cdot \tilde{q}_{+}\right)}{\tilde{q}_{+}^{2}}-\tilde{k}_{+}^{2},
$$

where $\tilde{k}_{+}=k-\frac{P_{+} \cdot k}{M_{\Omega}^{2}} P_{+}$and $\tilde{q}_{+}=q-\frac{P_{+} \cdot q}{M_{\Omega}^{2}} P_{+}[21]$.

For future discussion, it is worth mentioning that the function $b\left(\tilde{k}_{+}, \tilde{q}_{+}\right)$reduces to $-\frac{1}{2} \mathbf{k}^{2}\left(1-3 z^{2}\right)$, in the finalstate rest frame, where $z$ is the cosine of the angle between $\mathbf{k}$ and $\mathbf{q}$. We recover then the dependence on the spherical harmonic $Y_{20}(z) \propto\left(3 z^{2}-1\right)$ as in the nonrelativistic limit.

From (4.12)-(4.15), we can conclude that the state $D 3$ is responsible by the nonzero results of the electric quadrupole form factor and that both $D 3$ and $D 1$ contribute to the magnetic octupole form factor. In the limit $a=0$ (no $D 3$ state) and $b=0$ (no $D 1$ state), we recover the results of an $S$-state model with $G_{E 2} \equiv 0$ and $G_{M 3} \equiv 0[4,20,22]$.

In the expressions for the electromagnetic form factors (4.12)-(4.15), notice that the functions $G_{E 2}$ and $G_{M 3}$ include dependence on $\mathcal{I}_{D 3} / \tau$ and $\mathcal{I}_{D 1} / \tau$. These form factors are, however, well defined in the limit $\tau \rightarrow 0$ $\left(Q^{2} \rightarrow 0\right)$, since we can show that $\mathcal{I}_{D 3} \propto Q^{2}$ and $\mathcal{I}_{D 1} \propto$ $Q^{2}$ near $Q^{2}=0$ [21].
We can now discuss the normalization factor $N^{2}$. When the baryon elastic form factors are calculated in the first order of $a$ and $b$ and drop terms of the order of $a^{2}$ and $b^{2}$, one should take $N^{2}=1$. We notice, however, that in the limit $Q^{2}=0$ we can calculate the baryon charge using all orders of $a$ and $b$. In that case, we obtain $N^{2}=1 /\left(1+a^{2}+b^{2}\right)$. In the previous work, we choose to perform the calculations with $N^{2}=1 /\left(1+a^{2}+b^{2}\right)$ and include a theoretical band where the upper limit is determined by $N^{2}=1$. With this procedure, one obtains consistent results for $G_{E 0}(0)$ which must reproduce the $\Omega^{-}$ electric charge $\left[\mathcal{I}_{S} \rightarrow 1, G_{E 0}(0) \rightarrow \tilde{e}_{\Omega} \rightarrow-1\right]$.

In the present work, we simplify the previous procedure, assuming that the best estimate is the average between $N^{2}=1$ and $N^{2}=1 /\left(1+a^{2}+b^{2}\right)$. Our central value is then determined by

$$
N^{2}=\frac{1+\frac{a^{2}}{2}+\frac{b^{2}}{2}}{1+a^{2}+b^{2}} .
$$

To take into account the theoretical uncertainty, we use $N^{2}=1$ to the upper limit, as before, and $N^{2}=1 /(1+$ $\left.a^{2}+b^{2}\right)$ for the lower limit. The new procedure favors the fit to the data, since the fit to the $G_{E 0}$ lattice data at low $Q^{2}$ is improved when $N^{2}$ is closer to 1 .

\section{Large $Q^{2}$ behavior}

One can now look for the asymptotic form of the $\Omega^{-}$ electromagnetic form factors. The analysis of the overlap integrals $\mathcal{I}_{S}, \mathcal{I}_{D 3}$, and $\mathcal{I}_{D 1}$ shows that

$$
\mathcal{I}_{S}, \quad \mathcal{I}_{D 3}, \quad \mathcal{I}_{D 1} \propto \frac{1}{Q^{4}}
$$

apart logarithmic corrections. These asymptotic falloffs are valid for the parametrizations (4.2)-(4.4) and (4.6)-(4.8).

Taking the previous results into account, one can conclude from Eqs. (4.12)-(4.15) that the form factors are ruled by Eqs. (2.6) and (2.7) for large $Q^{2}: G_{E 0}, G_{M 1} \propto$ $1 / Q^{4}$ and $G_{E 2}, G_{M 3} \propto 1 / Q^{6}$, apart logarithmic corrections.

The results (4.21) are the consequence of the combination of the form of the radial wave functions in the overlap integrals and the fact that in the first order in the coefficients $a$ and $b$ all overlap integrals have at least a contribution of the $S$-state radial wave function. Since the overlap integrals are invariant, the integrals can be performed in any frame. The calculations are simplified when we choose the frame where the $S$ state is at rest. In these conditions, one can prove that if we use an $S$-state radial wave functions with the form $\psi_{S} \propto 1 /(\alpha+\chi)^{3}$ one obtain for the overlap integrals, falloffs with $1 / Q^{6}$, without logarithmic corrections. This result is derived in Appendix G from Ref. [83]. If we consider instead $\psi_{S} \propto 1 /(\alpha+\chi)^{2}$, one concludes that the overlap integrals are dominated by terms on $\frac{1}{Q^{4}}\left(\log \frac{Q^{2}}{M_{B}^{2}}\right)$, 
TABLE I. Adjustable parameters of the fits of the spacelike (SL) and timelike (TL) data. Included are the admixture coefficients $a$ and $b$ of the $D 3$ and $D 1$ states, and the parameters associated with the $S-\left(\alpha_{1}, \alpha_{2}\right), D 3-\left(\alpha_{3}\right)$, and $D 1$-state $\left(\alpha_{4}\right)$ radial wave functions, defined by Eqs. (4.2)-(4.4).

\begin{tabular}{lcccccc}
\hline \hline & $a$ & $b$ & $\alpha_{1}$ & $\alpha_{2}$ & $\alpha_{3}$ & $\alpha_{4}$ \\
\hline Fit SL data & 0.0322 & 0.2776 & 0.05927 & 0.1075 & 0.4437 & 0.5375 \\
Fit SL/TL data & 0.0304 & 0.2307 & 0.04250 & 0.1482 & 0.3340 & 0.2485 \\
\hline \hline
\end{tabular}

where $M_{B}$ is the mass of the baryon at rest (also demonstrated in Appendix G from Ref. [83]). In a present case, where the $S$-state radial wave function has a term $\psi_{S} \propto 1 /\left(\left(\alpha_{1}+\chi\right)\left(\alpha_{2}+\chi\right)\right)$, we can conclude that the overlap integrals are also dominated at large $Q^{2}$ with terms of the order $1 / Q^{4}$ with logarithmic corrections [118].

The possibility of verification of the condition (2.8) is discussed in Sec. V C. In that section, we analyze also the results obtained when we use the radial wave functions (4.6)-(4.8) and discuss the motivation to those expressions.

\section{ELECTROMAGNETIC FORM FACTORS IN THE TIMELIKE AND SPACELIKE REGIONS}

In the present section, we tested if the parametrizations discussed in the previous section are suitable to describe the available $\Omega^{-}$electromagnetic form factor data.

We divide the study in three steps:

(i) First, we tested if the simplest parametrizations, based on radial wave functions (4.2)-(4.4), defined by the asymptotic forms $G_{E 0}, G_{M 1} \propto 1 / Q^{4}$ and $G_{E 2}$, $G_{M 3} \propto 1 / Q^{6}$, are compatible with the lattice QCD data [spacelike (SL) region].

(ii) In a second step, we tested if the same kind of parametrization can also describe the timelike data, more specifically the data associated with the effective form factor $\left|G\left(q^{2}\right)\right|$ from CLEO [timelike (TL) region].

(iii) At the end, we tested if a modified model based on the radial wave functions (4.6)-(4.8) is consistent with the large- $Q^{2}$ condition (2.8) and debate the range of $Q^{2}$ where the relation can be fulfilled. [In the following, we use LQ2 to label the large- $Q^{2}$ condition (2.8)].

In a previous study [5], we considered only the lattice data in the region $Q^{2} \leq 1 \mathrm{GeV}^{2}$ for $G_{E 0}$ and $G_{M 1}$, from Ref. [7], because we were more focused in the low- $Q^{2}$ behavior of the form factors, including the results for
$G_{E 2}(0)$ and $G_{M 3}(0)$. In the present work, we extend the range to $Q^{2} \leq 2 \mathrm{GeV}^{2}$, in order to take into account large$Q^{2}$ effects on the form factors, which are pertinent to the timelike region. We discard the lattice QCD data for $Q^{2}>$ $2 \mathrm{GeV}^{2}$ because those simulations are affected by very large error bars and cannot be used to discriminate between different parametrizations.

Since Ref. [7] presents no data for $G_{M 3}$, we include in our database the $G_{M 3}$ data point from Boinepalli et al. [13] for $Q^{2}=0.23 \mathrm{GeV}^{2}$, even though the result is not very accurate.

\section{A. Adjust parameters to the spacelike data}

We adjust the free parameters of the model, the admixture coefficients $a, b$ and the parameters of the radial wave functions (4.2)-(4.4), $\alpha_{i}(i=1, \ldots, 4)$, to the spacelike data from our database. The value associated with the magnetic moment (2.1) is not included in the present fit because it was already used in the calibration of the strange quark current (fixes $\kappa_{s}$ ) [4]. The parameters of the best fit are presented in the first row of Table I (fit SL data).

The quality of the fit, estimated by the chi square per data point for the different subsets of data $\left(G_{E 0}, G_{M 1}, G_{E 2}\right.$, and $G_{M 3}$ ), is presented in the first row of Table II. In the column "Total," we present the total chi square per data point.

Compared with our previous study of the $\Omega^{-}$form factors from Ref. [5], we obtain a better description of the $G_{E 0}, G_{M 1}$ form factors and improve the overall description of the data (smaller total chi square per data point). There are three main reasons for this improvement: because we increase the range of the $Q^{2}$ lattice data and the chi square associated to the large $Q^{2}$ is smaller, because we consider a radial wave function for $\psi_{S}$ (4.2) with a falloff which better describe the data, and also because $\psi_{S}$ include two momentum range scales (an extra parameter). The most relevant factor to this improvement is the form of radial

TABLE II. The quality of the fit measured by the chi square per data point and percentages of $D 1$ and $D 3$ states. $|G|$ represents the timelike data. LQ2 labels the large- $Q^{2}$ condition (2.8).

\begin{tabular}{lcccccccc}
\hline \hline & $G_{E 0}$ & $G_{M 1}$ & $G_{E 2}$ & $G_{M 3}$ & $|G|$ & Total & $\% D 1$ & $\% D 3$ \\
\hline Fit SL data & 2.64 & 1.24 & 0.25 & 2.37 & & $\mathbf{1 . 5 4}$ & 0.096 & 7.15 \\
Fit SL/TL data & 2.53 & 1.66 & 0.37 & 3.40 & 2.79 & $\mathbf{1 . 7 4}$ & 0.088 & 5.05 \\
Fit SL/TL + LQ2 & 2.54 & 1.92 & 0.48 & 5.33 & 3.33 & $\mathbf{1 . 9 1}$ & 0.092 & 7.55 \\
\hline \hline
\end{tabular}



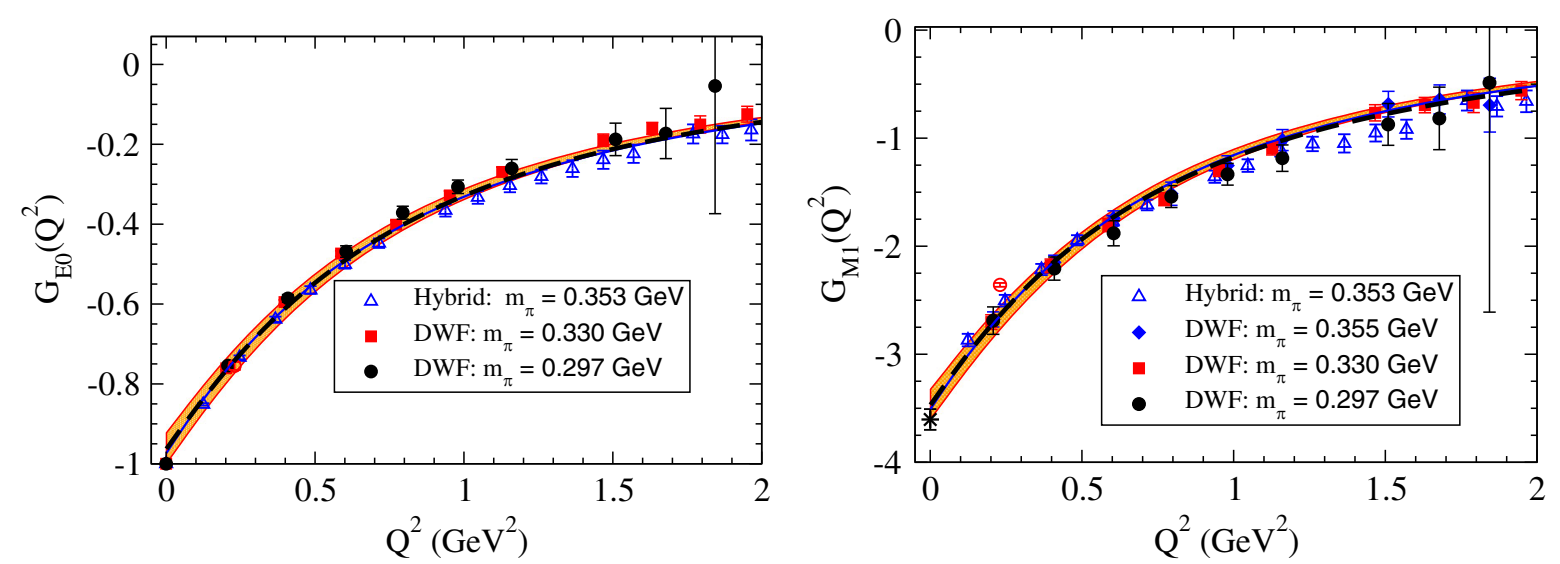

FIG. 1. Form factors $G_{E 0}$ and $G_{M 1}$. Fits to the data: SL (dashed line), SL/TL (solid line), and SL/TL + LQ2 (orange band). Lattice QCD data from Alexandrou et al. [7]. For $G_{M 1}$ we include also the experimental result $G_{M 1}(0)=-3.60 \pm 0.09$ [10] $(*)$. The open circles represent the result for $Q^{2}=0.23 \mathrm{GeV}^{2}$ from Boinepalli et al. [13].
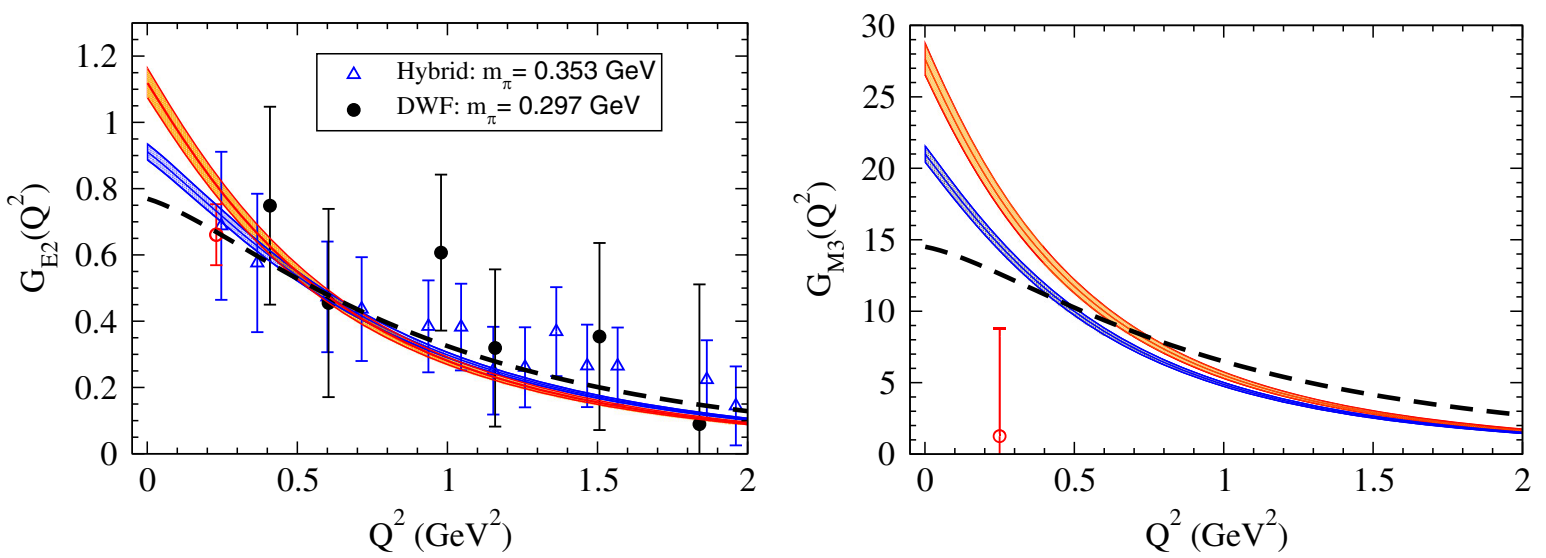

FIG. 2. Form factors $G_{E 2}$ and $G_{M 3}$ Fits to the data: SL (dashed line), SL/TL (blue solid line/blue band), and SL/TL + LQ2 (red solid line/orange band). Lattice QCD data from Alexandrou et al. [7]. The open circles represent the result for $Q^{2}=0.23 \mathrm{GeV}{ }^{2}$ from Boinepalli et al. [13].

wave function, since we replaced a tripole form $1 /\left(\alpha_{i}+\chi\right)^{3}$ in Ref. [5] by a product of two monopoles $1 /\left(\alpha_{i}+\chi\right)$. This conclusion was confirmed by numerical calculations.

The improvement in the description of the data with the new parametrization for $\psi_{S}$ is pertinent because it shows that a radial wave function compatible with the large- $Q^{2}$ pQCD behavior, Eqs. (2.6) and (2.7), can improve also the description of the low- $Q^{2}$ region. We emphasize that the use of two scales, $\alpha_{1}, \alpha_{2}$ in the radial wave function, instead of one global scale, $\alpha_{1}$, as in Ref. [5], also contributes to the improvement.

In Table II, we include also the relative contributions from the $D 1$ and $D 3$ states. We notice that the values of $a$ and $b$ are very close to the values of the previous work: $a=0.0341$ and $b=0.2666$ [5] (see Table I). It is then worth mentioning that, although based on different $S$-state radial wave functions, the mixtures of the $D 1$ and $D 3$ states, the fit from Ref. [5], and the new fit are very similar, with about $0.1 \%$ and $7 \%$ for the $D 3$ and $D 1$ states, respectively.
The conclusion that the $D 1$ state has a larger contribution is preserved. Notice, however, that the $0.1 \%$ of the state $D 3$ is essential to describe the electric quadrupole form factor data, according to Eq. (4.14).

We look now for the numerical results for the form factors for $G_{E 0}, G_{M 1}, G_{E 2}$, and $G_{M 3}$, represented in Figs. 1 and 2 by the dashed lines. We do not include the theoretical uncertainty band for clarity. As anticipated from the results for the chi squares, we obtain a good description of the $G_{M 1}$ and $G_{E 2}$ data. The $G_{E 0}$ lattice data are more difficult to describe, due to the behavior of the different datasets and the small error bars.

The comparison with the $G_{E 2}$ and $G_{M 3}$ lattice QCD data are presented in Fig. 2. The fit (dashed line) describes well the lattice QCD data for $G_{E 2}$ within the accuracy of the data points. The small value obtained for the $G_{E 2}$ partial chi square $(0.25)$ is the consequence of the large error bars on the data. As for $G_{M 3}$, the estimate can be compared only with the single data point 
$G_{M 3}\left(0.23 \mathrm{GeV}^{2}\right)=1.25 \pm 7.50$ [13]. The present result overestimates the data, but only by 1.1 standard deviations.

When compared with the estimate from Ref. [5], one has an increment of $13 \%$ for $G_{E 2}(0)$ and a reduction of $6 \%$ on $G_{M 3}(0)$. The falloffs of $G_{E 0}$ and $G_{M 3}$ with $Q^{2}$ are similar to the ones from Ref. [5].

\section{B. Adjust parameters to the spacelike and timelike data}

In the previous section, we demonstrated that the covariant spectator quark model is successful in the description of the $\Omega^{-}$spacelike data.

One can notice, however, that the test of the model is restricted to the range $0 \leq Q^{2} \leq 2 \mathrm{GeV}^{2}$. The model was not tested in the large- $Q^{2}$ region because the lattice $\mathrm{QCD}$ simulations are limited in the range of $Q^{2}$, and it was also not tested in the timelike region.

The next step is to test if the derived parametrization is consistent with the $\Omega^{-}$timelike data obtained from $e^{+} e^{-} \rightarrow$ $\Omega^{-} \bar{\Omega}^{+}$cross section data in CLOE [61], expressed in terms of the effective form factor $\left|G\left(q^{2}\right)\right|$. This test was performed with the model parametrization from Ref. [5] in Ref. [6]. The conclusion was that either the value of $G_{M 3}(0)$ is overestimated or the form factors drop off much faster that in the original parametrization.

The new timelike data provide then a unique opportunity to study the magnitude of $G_{M 3}(0)$ and the falloff of the $\Omega^{-}$ form factors, which cannot be tested by the available lattice QCD (limited in the range of $Q^{2}$ ).

To extend the calculations of the covariant spectator quark model to the timelike region $\left(q^{2}=-Q^{2}>0\right)$, we use the asymptotic relations proposed in Ref. [6] for the electric $(\ell=0,2)$ and magnetic $(\ell=1,3)$ form factors

$$
\begin{gathered}
G_{E \ell}^{\mathrm{TL}}\left(q^{2}\right)=G_{E \ell}\left(Q^{2}+2 M_{\Omega}^{2}\right), \\
G_{M \ell}^{\mathrm{TL}}\left(q^{2}\right)=G_{M \ell}\left(Q^{2}+2 M_{\Omega}^{2}\right),
\end{gathered}
$$

where the index TL indicates the timelike form factors. On the rhs, $G_{E \ell}$ and $G_{M \ell}$ represent the spacelike form factors. To calculate the effective form factors $\left|G\left(q^{2}\right)\right|$ of $\frac{3}{2}+$ baryons, we use the replacements (2.4) and (2.5) and the $G_{E \ell}$ and $G_{M \ell}$ spacelike form factors [69]. The relations (2.4) and (2.5) are derived from general physics and mathematical principles, including unitarity and the Phragmén-Lindelöf theorem, valid for analytic function of $q^{2}$ for very large $\left|q^{2}\right|[90,92]$. A consequence of the approximation is that the form factors are also real functions in the timelike region for large $q^{2}$. The shifts of $2 M_{\Omega}^{2}$ on the rhs of Eqs. (2.4) and (2.5) are motivated by the difference between the spacelike $\left(Q^{2}=0\right)$ and timelike thresholds $\left(q^{2}=4 M_{\Omega}^{2}\right)$. The relations used here include then finite $q^{2}$ corrections to the asymptotic limit [6].

The parameters associated with the global fit are presented in the last row of Table I. The corresponding chi square per data point for each form factor, is included in the second row of Table II (fit SL/TL data). The column $|G|$ indicates the chi square associated with the timelike data.

An interesting result from Table II is that the consideration of the timelike data leads to the improvement of the description of the $G_{E 0}$ data, meaning that the falloff of $G_{E 0}$ is relevant for the description of the timelike data. The differences between the two parametrizations (SL or SL/ TL) are related to the variation in about $30 \%$ on $\alpha_{1}$, as one can see in Table I. Recall that $G_{E 0}$ depend exclusively on the $S$ state. These differences, however, are not perceived on the graph for $G_{E 0}$ below $Q^{2}=2 \mathrm{GeV}^{2}$.

The comparison of the global fit (SL/TL) with the spacelike data is presented in Figs. 1 and 2. In Fig. 1, we include only the blue solid line for clarity. The theoretical uncertainty associated with the blue line has a magnitude similar to the orange band, discussed in the next subsection. In Fig. 2, we present the result of the fit by the blue band in order to include the theoretical error associated with the normalization factor $N^{2}$, as discussed in Sec. IV C.

The results of the fit to the SL and TL data for $G_{E 0}$ and $G_{M 1}$ (solid line) are very similar to the results of the fit to the SL data (dashed line). We conclude then that the main differences between the two fits (SL and SL/TL) appear only for values of $Q^{2}$, larger than $2 \mathrm{GeV}^{2}$.

The results for $G_{E 2}$ and $G_{M 3}$ displayed in Fig. 2 are more relevant. One can notice the increasing of $G_{E 2}$ and $G_{M 3}$ near $Q^{2}=0$ and a stronger falloff with $Q^{2}$ in comparison with the fit which ignore the timelike data. The quality of the description of the $G_{E 2}$ data are not substantially modified (large error bars, partial chi square per data point of $0.2-0.4$ ). As for $G_{M 3}$, the impact of the timelike data is more significant. Notice that the quality of the description of the $G_{M 3}$ data point is reduced in comparison with the previous fit (dashed line). The estimate differs from the data by 1.8 standard deviations. When we take into account the timelike data, we deteriorate the description of the $G_{M 3}$ data, represented by the point with $Q^{2}=0.23 \mathrm{GeV}^{2}$.

The main difference in the new solution to $G_{M 3}$ is in the faster falloff with $Q^{2}$, indicating that the magnitude of the function is significantly reduced for large $Q^{2}$, contributing to a significant suppression of the term $\left|G_{M}\right|^{2}$ in $\left|G\left(q^{2}\right)\right|$ [see Eq. (2.5)].

The results for the effective form factor $\left|G\left(q^{2}\right)\right|$ for the $\Omega^{-}$are presented in Fig. 3 and compared with the more recent data from CLOE for $q^{2}=14.2$ and $17.4 \mathrm{GeV}^{2}$ [61]. We omitted the first measurement at $q^{2}=14.2 \mathrm{GeV}^{2}$ from CLOE [12], since it is superseded by a more recent analysis [61]. The dashed line corresponds to our best estimate based on Eqs. (5.1) and (5.2) and the spacelike and timelike data. The thin dashed lines indicate the theoretical error based on finite correction on $q^{2}$ for the asymptotic relations between spacelike and timelike regimes, which are valid strictly when $q^{2} \rightarrow \pm \infty$. We estimate these errors using the replacements on Eqs. (5.1) and (5.2): $Q^{2}+2 M_{\Omega}^{2} \rightarrow Q^{2}$ for 


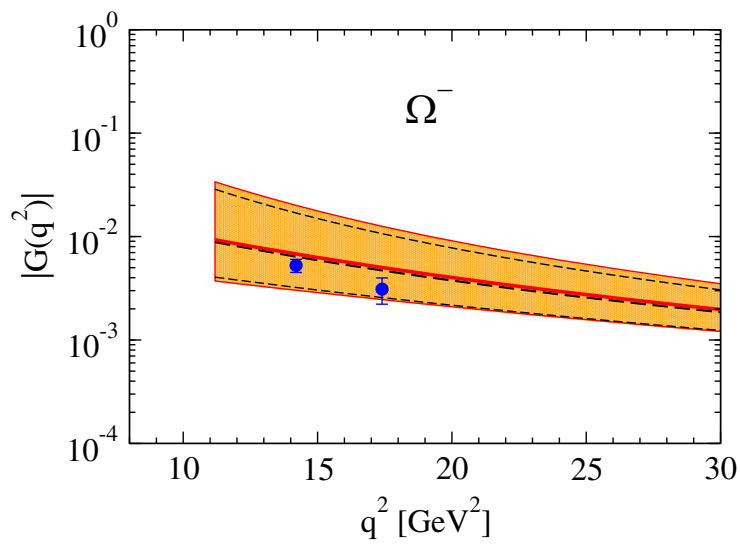

FIG. 3. Results for the $\Omega^{-}$effective form factor $\left|G\left(q^{2}\right)\right|$ in the timelike region $\left(q^{2}>0\right)$. The thick dashed line indicates the result of the fit SL/TL which ignore the large- $Q^{2}$ constraints, and the thin dashed lines represent the upper and lower limits (theoretical error). The solid line is the best estimate (fit $\mathrm{SL} / \mathrm{TL}+\mathrm{LQ} 2$ ) based on asymptotic relations, including the theoretical error (orange band). The data are from CLEO [61].

the upper limit and $Q^{2}+2 M_{\Omega}^{2} \rightarrow Q^{2}+4 M_{\Omega}^{2}$ for the lower limit [6]. At very large $Q^{2}$, the width of the variation become negligible and the leading-order falloff of $G, 1 / q^{4}$, emerges [6].

We return to this discussion after taking into account the impact of the large- $Q^{2}$ relation (2.8) on the structure of the form factors.

\section{Model compatible with the asymptotic conditions}

In Sec. IV D, we conclude that the leading-order dependence of the electromagnetic form factors at large $Q^{2}: G_{E 0}, G_{M 1} \propto 1 / Q^{4}$ and $G_{E 2}, G_{M 3} \propto 1 / Q^{6}$, is naturally reproduced with the radial wave functions (4.2)-(4.4). One can then ask if the large- $Q^{2}$ constraint (2.8) can also be accomplished with the same model for the radial wave functions. The answer is no, as demonstrated below.

For the following discussion, it is necessary to know that $\tilde{f}_{\Omega}$ is a negative function of $Q^{2}$ (see Appendix B) and the signs of the overlap integrals $\mathcal{I}_{S}$ and $\mathcal{I}_{D 1}$ defined by (4.16) and (4.18). Based on the parametrizations discussed in the previous subsections, we assume also that the mixture coefficients $a$ and $b$ are positive, as suggested by the previous analysis (SL and ST/TL fits).

The integral $\mathcal{I}_{S}$ is positive by construction (positive integrate functions), and consequently, it does not change the sign. The integrals $\mathcal{I}_{D 3}$ and $\mathcal{I}_{D 1}$ include the angular function $Y_{20}(z) \propto\left(3 z^{2}-1\right)$, when we consider the finalstate rest frame. In the limit $Q^{2}=0$, the integrals $\mathcal{I}_{D 3}$ and $\mathcal{I}_{D 1}$ vanish due to the factor $Y_{20}(z)$. For finite $Q^{2}$, the $D$ state integrals can be positive or negative depending on $Q^{2}$ and on the square momentum parameters of the radial wave functions $\left(\alpha_{i}, i=1, \ldots, 4\right)$. For the typical values of the parameters (determined from the fits to the $0 \leq Q^{2} \leq$ $2 \mathrm{GeV}^{2}$ region), one concludes that the functions $\mathcal{I}_{D 1}$, as $\mathcal{I}_{D 3}$, do not change sign in wide a range of $Q^{2}$. Numerical calculations suggest that the possible zeros for $\mathcal{I}_{D 3}$ and $\mathcal{I}_{D 1}$ appear only for $Q^{2}>10^{6} \mathrm{GeV}^{2}$, well above the present-day range of experiments. Thus, for the purpose of the applications of the parametrizations (4.2)(4.4), we can assume that $\mathcal{I}_{D 3}$ and $\mathcal{I}_{D 1}$ are functions with a defined sign (do not change sign).

We can now explain why the parametrizations (4.2)(4.4) are incompatible with the relation (2.8). When we combine Eqs. (4.13) and (4.15) with the condition (2.8), we conclude that the last condition is valid if $\mathcal{I}_{S}=2 b \mathcal{I}_{D 1}$ (see Appendix B). A consequence of the previous relation is that the contribution from the D3 state is not relevant for Eq. (2.8).

The verification of Eq. (2.8) implies that $G_{M 1}$ and $G_{M 3}$ must have the same sign for large $Q^{2}$. The $S$-state gives the dominant positive contribution to $G_{M 1}<0$, while the state $D 1$ gives a large contribution to $G_{M 3}>0$, near $Q^{2}=0$, implying that $b \mathcal{I}_{D 1}<0$, since $\tilde{f}_{\Omega}<0$. The conclusion is then that $\mathcal{I}_{S}>0$ and $b \mathcal{I}_{D 1}<0$ at low $Q^{2}$. Since, as mentioned, the large- $Q^{2}$ relation is equivalent to $\mathcal{I}_{S}=$ $2 b \mathcal{I}_{D 1}$, and $\mathcal{I}_{S}>0$ does not change sign, the inference is that $b \mathcal{I}_{D 1}$ should change sign at large $Q^{2}$ in order to satisfy (2.8). We recall, however, as discussed, that $b \mathcal{I}_{D 1}$ does not change sign in the present range of study.

The corollary of the previous discussion is that the parametrizations (4.2)-(4.4) are incompatible with (2.8), when the parameters of the radial wave functions are determined by available lattice QCD data. We can enforce the verification of the condition (2.8), but then we fail to obtain an accurate description of the spacelike form factor data.

To ensure the validity of Eq. (2.8), we consider then the parametrizations (4.6)-(4.8). The new expression for $\psi_{S}$ is compatible with a change of sign on the overlap integral $\mathcal{I}_{S}$. We modify also the expressions for $\psi_{D 3}$ and $\psi_{D 1}$ including a factor common to $\psi_{S}$. The expression for $\psi_{D 1}$ is motivated by the relation between the overlap integrals $\mathcal{I}_{S}$ and $\mathcal{I}_{D 1}$. As for $\psi_{D 3}$, we use a dependence analog to $\psi_{D 1}$ for consistency with the $D$-state structure. The parametrizations (4.6)-(4.8) are also compatible with the relations (4.21) [119]. The new form for $\psi_{S}$ can be used to induce a change of sign in the function $G_{M 1}$ and to ensure that $G_{M 1}$ and $G_{M 3}$ have the same sign at large $Q^{2}$, according to $\mathcal{I}_{S}=2 b \mathcal{I}_{D 1}$. The condition $\mathcal{I}_{S}=2 b \mathcal{I}_{D 1}$ may be difficult to impose analytically but can be approximated numerically within a certain accuracy for a given region of $Q^{2}$, centered on a given large scale $\bar{Q}^{2}$, with a particular choice of parameters.

To the best of our knowledge, this is the first time that the relation (2.8) is considered in the context of the $\frac{3}{2}+$ baryon form factors, in general, and the $\Omega^{-}$form factors in particular.

We tested tentatively if there was a scale $\bar{Q}^{2}$ where the large $Q^{2}$ condition (2.8) could be satisfied in the interval 
TABLE III. Adjustable parameters of the fits to the spacelike and timelike (SL/TL) data with the large- $Q^{2}$ (LQ2) constraint (2.8), based on the parametrization of the radial wave functions (4.2)-(4.4). $S$ state $\left(r_{S}, \alpha_{1}^{\prime}, \alpha_{2}^{\prime}, \alpha_{1}, \alpha_{2}\right), D 3$ state $\left(\alpha_{1}, \alpha_{2}, \alpha_{3}\right)$, and $D 3$ state $\left(\alpha_{1}, \alpha_{2}, \alpha_{4}\right)$.

\begin{tabular}{|c|c|c|c|c|c|c|c|c|c|}
\hline & $a$ & $b$ & $\alpha_{1}^{\prime}$ & $\alpha_{2}^{\prime}$ & $r_{S}$ & $\alpha_{1}$ & $\alpha_{2}$ & $\alpha_{3}$ & $\alpha_{4}$ \\
\hline Fit SL/TL + LQ2 & 0.0316 & 0.2859 & 11.141 & 0.0818 & $1.845 \times 10^{-3}$ & 0.2018 & 0.1494 & 0.0789 & 1.811 \\
\hline
\end{tabular}

$\left[\bar{Q}^{2}-\Delta Q^{2}, \bar{Q}^{2}+2 \Delta Q^{2}\right]$, for a given $\Delta Q^{2}$, with an accuracy better than $\epsilon$. We consider the upper limit $Q_{p}^{2}=$ $\bar{Q}^{2}+2 \Delta Q^{2}$, with a difference $2 \Delta Q^{2}$ for $\bar{Q}^{2}$ to ensure a smoother convergence in the range between $Q_{m}^{2}=\bar{Q}^{2}-$ $\Delta Q^{2}$ and $\bar{Q}^{2}+\Delta Q^{2}$.

To include the condition (2.8) in our fit, we use the function

$$
\mathcal{R}\left(Q^{2}\right)=\frac{G_{M 1}\left(Q^{2}\right)-\frac{4}{5} \tau G_{M 3}\left(Q^{2}\right)}{G_{M 1}\left(Q^{2}\right)} .
$$

If the condition (2.8) is valid, we should have $\mathcal{R}\left(Q^{2}\right) \simeq 0$ for values of $Q^{2}$ near a large scale $\bar{Q}^{2}$.

We need then to test numerically if $\left|\mathcal{R}\left(Q^{2}\right)\right|$ is smaller than a given value in an interval $\left[Q_{m}^{2}, Q_{p}^{2}\right]$. Recall, however, that one expects also that $\mathcal{R}\left(Q^{2}\right) \propto 1 / Q^{2}$, for large $Q^{2}$, since $\left(G_{M 1}-\frac{4}{5} \tau G_{M 3}\right) \propto 1 / Q^{6}$ and $G_{M 1} \propto 1 / Q^{4}$, apart logarithmic corrections. To take into account the falloff $\mathcal{R}\left(Q^{2}\right) \propto 1 / Q^{2}$, we consider the condition

$$
\left|\mathcal{R}\left(Q^{2}\right)\right|<\frac{Q^{2}}{Q_{p}^{2}} \epsilon
$$

for $Q_{m}^{2}<Q^{2}<Q_{p}^{2}$. Thus, in the upper limit, one requires that $\left|\mathcal{R}\left(Q_{p}^{2}\right)\right|<\epsilon$. For $Q^{2}<Q_{p}^{2}$, however, one demands a softer condition in order to obtain a smooth convergence of $G_{M 1}$ to $\frac{4}{5} \tau G_{M 3}$ in the point $Q^{2}=Q_{p}^{2}$, with the required precision.

When we implement the constraints described above, the solutions (fits) compatible with the relation (2.8) are characterized by the values of $\bar{Q}^{2}, \Delta Q^{2}$, and $\epsilon$. To obtain a significant range of convergence, we choose $\Delta Q^{2}=$ $100 \mathrm{GeV}^{2}$. Once $\bar{Q}^{2}$ and $\Delta Q^{2}$ are defined, the value of $Q_{p}^{2}$ corresponds to the upper limit of the parametrization.

We vary then the value of $\bar{Q}^{2}$ looking for solutions with an accuracy better than $\epsilon$. The numeric calculations indicate that a fair description of the data $\left(\chi^{2} \leq 2\right)$ with a $1 \%$ accuracy $(\epsilon=0.01)$ is obtained for $\bar{Q}^{2}=900$ and $1000 \mathrm{GeV}^{2}$. Solutions with $\epsilon=0.02$ (2\% accuracy) can be obtained in a wider region of $\bar{Q}^{2}$, but the convergence between the two functions is not so smooth. We consider then the solutions with $\epsilon=0.01$.

We choose the solution with $\bar{Q}^{2}=900 \mathrm{GeV}^{2}$, since it provides the lowest value for chi square (best description of the overall data). Solutions with large $\bar{Q}^{2}$ tend to provide a better description of the $\left|G\left(q^{2}\right)\right|$ data and to increase the values of $G_{M 3}$ at low $Q^{2}$ (a less accurate description of the $G_{M 3}$ data). As one of the motivations of the present work is to investigate if there are solutions based on the our formalism compatible with the present $\Omega^{-}$electromagnetic form factor data and with the condition (2.8), we do not try to fine tune the value of $\bar{Q}^{2}$. In principle, better solutions (lower chi-square values) can be obtained varying $\bar{Q}^{2}$ near $900 \mathrm{GeV}^{2}$. Those solutions are, however, very similar to the selected one, and qualitatively equivalent to the case $\bar{Q}^{2}=900 \mathrm{GeV}^{2}$. Future experiments can provide further constraints on the parametrizations of the $\Omega^{-}$form factors and help to decide the appropriated scale for $\bar{Q}^{2}$.

In the context of our formalism, the parametrizations (4.6)-(4.8) are interpreted as effective corrections to the radial wave functions of the $\Omega^{-}$baryon, which resemble some properties of $\mathrm{pQCD}$ in a simplified form.

The parameters associated with the global fit of the spacelike and timelike data, constrained by the relation (2.8), are presented in Table III. The values of chi square per data point are presented in the last row of Table II (fit SL/TL + LQ2).

The best fit to the data, including the band of variation estimated from the values of $N^{2}$, is represented in Figs. 1 and 2 by the orange band. The more significant differences to the previous fits can be observed in Fig. 2 for $G_{E 2}$ and $G_{M 3}$. We recall that, based on the previous discussion, the upper limit of the present estimates is $Q_{p}^{2}=1100 \mathrm{GeV}^{2}$.

The function $G_{E 2}$ (orange band) is enhanced at low $Q^{2}$ compared to the previous fits. At $Q^{2}=0$, we obtain $G_{E 2}(0)=1.12 \pm 0.04$, a larger value than the estimate obtained by the SL/TL fit, $G_{E 2}(0)=0.91 \pm 0.02$, and almost twice the estimate from Ref. [5]. We recall that different extrapolations to $Q^{2}=0$ from the $G_{E 2}$ data, are expected due to the large uncertainty of the data. We avoid a detailed comparison of the results for $G_{E 2}$ with the literature, since our results are determined by the global fit to the lattice QCD data, presented in the left panel of Fig. 2.

As for the function $G_{M 3}$, we obtain a larger estimate for $G_{M 3}(0)$ with $G_{M 3}(0)=27.6 \pm 1.1$ (orange band). The solution for $G_{M 3}$ is also characterized by a strong falloff with $Q^{2}$, which contributes to a reduction of the magnetic contribution to the effective form factor $\left|G\left(q^{2}\right)\right|$ at large $q^{2}$.

The result for the effective form factor $\left|G\left(q^{2}\right)\right|$ is represented in Fig. 3 by the solid line within the range 


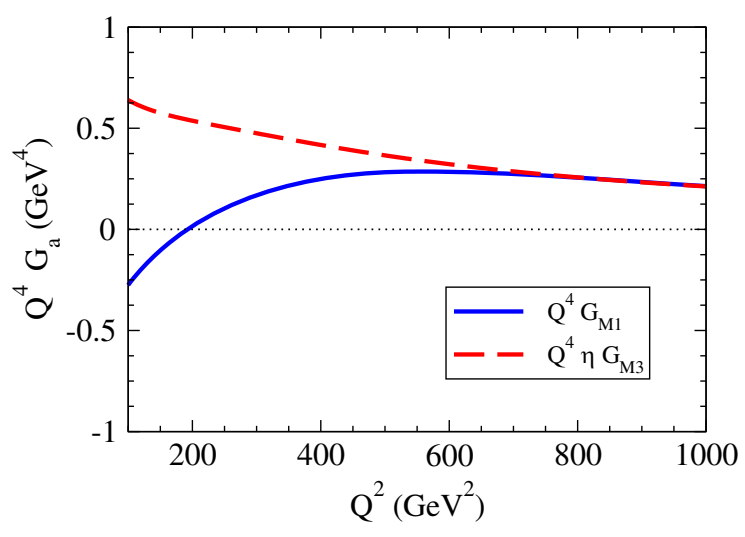

FIG. 4. Test of the relation (2.8) for large values of $Q^{2}$. We use $\eta=\frac{4}{5} \tau$.

represented by the orange band. This estimate is very close to the parametrization which does not take into account the large- $Q^{2}$ constraint (represented by the dash lines). Overall, one can say that the estimates based on the covariant spectator quark model are in good agreement with the large- $q^{2}$ data from CLOE within the theoretical errors of the asymptotic estimate.

Since the results of the last fit (SL/TL + LQ2) depend on the accuracy of the relation (2.8), we tested the convergence in the selected region (800-1000 $\mathrm{GeV}^{2}$ ). In Fig. 4, we present the comparison of $G_{M 1}$ and $\frac{4}{5} \tau G_{M 3}$ multiplied by $Q^{4}$. The factor $Q^{4}$ is included in order to remove the effects of the leading-order dependence of the form factors, dominated by terms of the order $1 / Q^{4}$. The results confirm that the relations are valid in good approximation (smooth convergence) in the range $700-1000 \mathrm{GeV}^{2}$.

The deviation from a horizontal line indicates that, even in the range $Q^{2}=700-1000 \mathrm{GeV}^{2}$, logarithmic corrections or terms of the order $1 / Q^{6}$ are still meaningful. We checked numerically that the form of the present parametrizations for the radial wave functions is consistent with a very slow falloff of the correction to the leading order

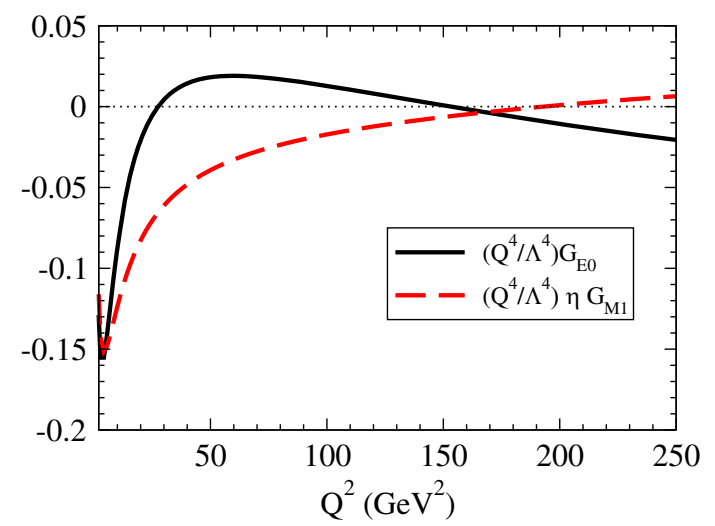

$1 / Q^{4}$. We do not attempt to check the exact scaling at very large $Q^{2}$ because the scale $Q^{2}=1100 \mathrm{GeV}^{2}$ is interpreted as the upper limit of our calculations and also because we are well above the range of the present day experiments, from which our model parametrizations are calibrated.

To have an idea about the general behavior of the form factors above the region displayed on Figs. 1 and 2, we represent in Fig. 5 the four form factors for $Q^{2} \geq 2 \mathrm{GeV}^{2}$. We multiply the functions by $\left(\frac{Q^{2}}{\Lambda^{2}}\right)^{2}$ with $\Lambda^{2}=2 \mathrm{GeV}^{2}$ in order to suppress (part of) the falloff of the form factors for large $Q^{2}$. With this representation, we complement the results from Figs. 1 and 2 , since $\left(\frac{Q^{2}}{\Lambda^{2}}\right)^{2}=1$, at the threshold of the representation. The inflection points near the threshold in the graph for $G_{E 0}$ and $G_{M 1}$ are the consequence of the factor $Q^{4}$ and are not relevant for the present discussion. The zero for the functions $G_{E 0}$ and $G_{E 2}$ for $Q^{2}=28 \mathrm{GeV}^{2}$ is a consequence of the of the zero of the function $\tilde{g}_{\Omega}$ defined by Eq. (4.10) and the particular parametrization of the strange quark form factors $f_{i 0}$, from Eqs. (3.5) and (3.6), determined in Ref. [4] by the study of the decuplet baryon form factors. This result is independent of the overlap integrals $\mathcal{I}_{S}$ and $\mathcal{I}_{D 3}$. Above $Q^{2}=28 \mathrm{GeV}^{2}, G_{E 2}$ became negative but almost negligible. The change of signs on $G_{E 0}$ for $Q^{2} \simeq 153 \mathrm{GeV}^{2}$ and of $G_{M 1}$ for $Q^{2} \simeq 193 \mathrm{GeV}^{2}$ is the consequence of the parametrizations (4.6)-(4.8), more specifically of the decomposition of $\psi_{S}$ into two terms. The changes of sign on $G_{M 1}$ are necessary for the validity of the relation (2.8), as discussed already.

In the case the zero on functions $G_{E 0}$ and $G_{E 2}$ is confirmed by future experiments, the zero is justified by a mechanism similar to the one associated with the zero of the proton electric form factor $G_{E p}=F_{1 p}-\frac{Q^{2}}{4 M_{N}^{2}} F_{2 p}$ $[81,120,121]$, where there is a competition between the two terms (Dirac $F_{1 p}$ and Pauli $F_{2 p}$ form factors).

Only new data (empirical or lattice) can confirm if our parametrization of the strange quark form factors, calibrated at low $Q^{2}$ [4], can be extended very large values of $Q^{2}$.

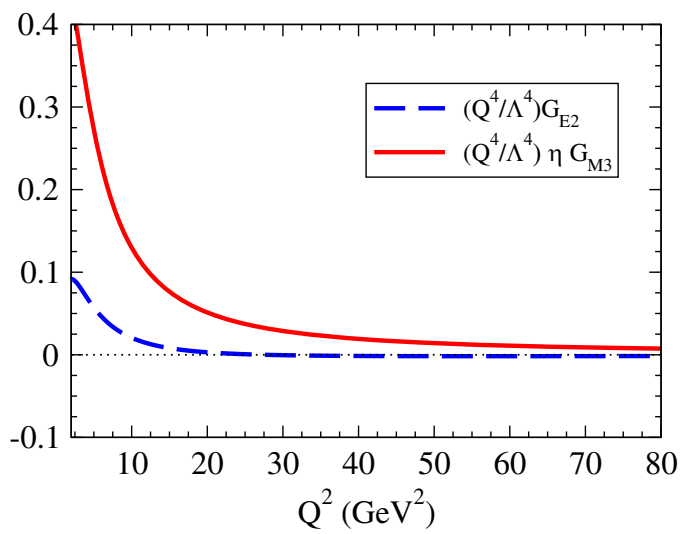

FIG. 5. Representation of the form factors $G_{E 0}, G_{M 1}, G_{E 2}$, and $G_{M 3}$ for large $Q^{2}$, calibrated by the factor $\left(\frac{Q^{2}}{\Lambda^{2}}\right)^{2}$, with $\Lambda^{2}=2$ GeV ${ }^{2}$ (spacelike region). We use also $\eta=\frac{1}{4}$ to reduce the scale of $G_{M 1}$ and $G_{M 3}$. Notice the zeros of the functions $G_{E 0}, G_{M 1}$ and $G_{E 2}$. 


\section{Discussion of the results}

From the results for the parametrizations which describe the SL/TL data and the parametrizations which verify also the LQ2 condition, one can conclude that it is possible to derive parametrizations which take into account the present knowledge of the $\Omega^{-}$form factors in the spacelike region (mainly lattice QCD data for $G_{E 0}, G_{M 1}$, and $G_{E 2}$ ) as well as the timelike data (effective form factor data).

We notice, however, that the description of the function $G_{M 3}$ is not as accurate as the others $\left(G_{E 0}, G_{M 1}\right.$, and $\left.G_{E 2}\right)$ and that the description of the effective form factor $\left|G\left(q^{2}\right)\right|$ is also not as perfect as the first three multipole form factors.

The limitations in the description of the functions $G_{M 3}$ and $\left|G\left(q^{2}\right)\right|$ are, however, also related to the limited weight of those functions in the fit. Notice that one has 100 spacelike data points for $G_{E 0} G_{M 1}$, and $G_{E 2}$; only one point for $G_{M 3}$; and two points for $\left|G\left(q^{2}\right)\right|$. The fit is then dominated by the 100 points of the first three form factors $\left(G_{E 0} G_{M 1}\right.$ and $\left.G_{E 2}\right)$. In principle, we could enhance the impact of the points $G_{M 3}$ and $\left|G\left(q^{2}\right)\right|$ including an extra weight in the fit (equivalent to a reduction on the error bars), but the procedure goes against the principle of giving the same treatment to the spacelike and the timelike regions (equal weight to spacelike and timelike points).

From the comparison between the two global fits (with and without LQ2), one can conclude that the consideration of the large- $Q^{2}$ relation (2.8) decreases the accuracy of the final fit, at the expenses of a less accurate description of $G_{M 3}$, as can be observed in Table II (compare values of the column $G_{M 3}$ ). Our estimate of $G_{M 3}$ deviates from the lattice data by 2.3 standard deviations (see Fig. 2).

One can notice, however, that the estimate of $G_{M 3}$ depends exclusively on a unique point estimated in 2009, affected with a large uncertainty. The lattice QCD techniques used in the past can be used today to estimate $G_{M 3}$ for a larger set of $Q^{2}$ points with a better accuracy. New lattice QCD simulations of $G_{M 3}$ for values of $Q^{2}$ closer to $Q^{2}=0$ can then be used to test our estimate for $G_{M 3}(0)$ and the shape of $G_{M 3}$ at low $Q^{2}$.

Our best parametrization (SL/TL + LQ2) suggests then that our estimate for $G_{M 3}(0)$ is most likely incompatible with the available lattice result for $G_{M 3}\left(0.23 \mathrm{GeV}^{2}\right)$. The large values of the function $G_{M 3}$ are a consequence of the consideration of the timelike data and the large- $Q^{2}$ condition (2.8). It is interesting to notice, however, that our large estimate for $G_{M 3}(0)$ is comparable with other theoretical estimates presented in the literature as discussed next.

In the literature, there are only a few estimates of the function $G_{M 3}$. In chiral perturbation theory, the magnetic octupole momentum vanishes at the next-to-leading order of the chiral expansion [50]. Also, the chiral solition model approaches give null results for the function $G_{M 3}$ [55].
TABLE IV. Results for $G_{M 3}(0)$ and magnetic octupole in units $10^{-3} \mathrm{fm}^{3}$. An alternative representation of the magnetic octupole uses units $e \mathrm{fm}^{3}$, where is the elementary electric charge $(e=\sqrt{4 \pi \alpha} \simeq 0.303)$. Noncovariant $\mathrm{QM}$ (sym) indicates a $S U(3)$ symmetrical model.

\begin{tabular}{lcc}
\hline \hline & $G_{M 3}(0)$ & $\mathcal{O}_{\Omega}\left(10^{-3} \mathrm{fm}^{3}\right)$ \\
\hline QCD sum rules [52] & $64.3 \pm 16.1$ & $16.0 \pm 4.0$ \\
Non covariant QM (sym) [56] & 48.2 & 12.0 \\
Noncovariant QM [56] & 12.2 & 3.04 \\
Spectator [5] & 15.5 & 3.85 \\
Spectator SL & $14.4 \pm 0.5$ & $3.61 \pm 0.14$ \\
Spectator SL/TL & $21.0 \pm 0.6$ & $5.22 \pm 0.14$ \\
Spectator SL/TL + LQ2 & $27.6 \pm 1.1$ & $6.88 \pm 0.27$ \\
\hline \hline
\end{tabular}

Calculations based on the Dyson-Schwinger equations formalism [57,58] suggest that the magnitude of $G_{M 3}$ may be small but the sign is uncertain. Reference [57] estimates that $G_{M 3}(0) \simeq+0.5$, while the calculations from Ref. [58] point to $G_{M 3}(0) \simeq-0.3$ or $G_{M 3}(0) \simeq-0.5$. The Dyson-Schwinger results of the function $G_{M 3}$ are sensitive to the truncation used in the interaction kernels [58].

In Table IV, we compare our estimates with calculations based on QCD sum rules [52] and with the $S U(3)$ noncovariant quark model [56]. The first estimate from Ref. [56] is based on an exact $S U(3)$ model (symmetric wave function); the second estimate considers the breaking of $S U(3)$. From the observation of the table, we can conclude that our estimate is comparable in magnitude with the estimates based on QCD sum rules and the symmetrical noncovariant quark model. Table IV includes also the results for the octupole magnetic moment for an easy comparison with other works.

The previous analysis shows that the estimate of the octupole magnetic moment is an open problem, and more theoretical and experimental efforts are necessary to clarify the situation. Also for that reason, lattice QCD simulations for $G_{M 3}$ at the physical mass of the $\Omega^{-}$are mandatory. Accurate results for $G_{M 3}$ can be used to extrapolate $G_{M 3}(0)$, following the lines of previous estimates of $G_{E 2}(0)$ based on lattice QCD data [5,7].

One can also discuss the shape of $\Omega^{-}$based on the electric quadrupole $\left[\propto G_{E 2}(0)\right]$ and magnetic octupole $\left[\propto G_{M 3}(0)\right]$ moments, which measure deviations from the electric charge and magnetic dipole distributions from a spherical form. For negative charge baryons, positive moments indicate a compression along the polar axis (oblate shape), and negative moments indicate an elongation in the polar axis (prolate shape), since the charge density depends on the baryon charge $[19,22,85]$. We conclude, then, that the $\Omega^{-}$presents a distribution of electric charge and magnetic dipole compressed along the spin axis, corresponding to an oblate shape. The shape of the $\Omega^{-}$resembles then the shape of the $\Delta^{+}$[positive charge, $G_{E 2}(0)<0$ and $G_{M 3}(0)<0$ ], according to the 
covariant spectator quark model estimates [21], ignoring the scale of the deformation.

Based on our results for $G_{E 0}$, we estimate the size of the $\Omega^{-}$given by the electric charge square radius as $r_{E 0}^{2} \simeq 0.24 \mathrm{fm}^{2}$. This result corresponds to about one-third of the value measured for the proton $\left(r_{E p}^{2}=0.707 \mathrm{fm}^{2}\right)$ [10], suggesting that $\Omega^{-}$is much more compact than the proton.

Concerning the results for the function $\left|G\left(q^{2}\right)\right|$, in the timelike region, we conclude that the estimates which exclude and include the condition (2.8) are very similar (see Fig. 3). The main differences between the two parametrizations appear for the function $G_{M 3}$, providing an additional motivation for performing lattice QCD simulations for this form factor.

Two final remarks about about our calculations in the timelike region and the range of validity of the model calculations are in order.

The $\left|G\left(q^{2}\right)\right|$ data from CLEO used in our calibrations correspond to the values of $q^{2}=14.2$ and $17.4 \mathrm{GeV}^{2}$. These values for $q^{2}$ are still very close to the threshold of the $e^{+} e^{-} \rightarrow \Omega^{-} \bar{\Omega}^{+}$reaction $\left(q^{2}=4 M_{\Omega}^{2} \simeq 11.2 \mathrm{GeV}^{2}\right)$. Therefore, the use of an expression valid for very large $\left|Q^{2}\right|$ may not be fully justified. Since theses are the only available data for $q^{2}>0$, we used the data anyway.

The second remark is that, for simplicity, we did not take into account the uncertainty associated with our estimate of $\left|G\left(q^{2}\right)\right|$, in the calibration of the model in the calculation of the chi square. If we take into account those uncertainties (integrating out on a normal distribution centered on the model result for $\left|G\left(q^{2}\right)\right|$ ), we improve the quality of the description of this function, since the data are inside the one-standard-deviation region. In that case, we would obtain a smaller value for the $|G|$ chi square, and consequently a smaller global chi square per data point. We avoid this procedure because, as mentioned above, the theoretical estimates are still far way from the large- $\left|Q^{2}\right|$ region, where the estimate and the estimate of error are valid, and also because we do not want to reduce at this stage the impact of the timelike data in the calibration of the model.

Future measurements for larger $q^{2}$ values, farther away from the threshold $4 M_{\Omega}^{2}$, are of capital importance to check if the present falloffs associated to $\left|G\left(q^{2}\right)\right|$ and $G_{M 3}$ are correct or if the present shape of the function $G_{M 3}$ has to be corrected.

\section{E. Predictions of $|G|$ for large $\boldsymbol{q}^{\mathbf{2}}$}

Our final parametrization, which takes into account the timelike region and the large- $Q^{2}$ relation (2.8), can now be used to make predictions for effective form factor $\left|G\left(q^{2}\right)\right|$, for larger values of $q^{2}$. Recall that the function $\left|G\left(q^{2}\right)\right|$ is extracted from $e^{+} e^{-}$collision experiments cross sections,
TABLE V. Estimates of $\left|G\left(q^{2}\right)\right|$ for large $q^{2}$, compatible with condition (2.8). The values between comas represent the average of the upper and lower limits. Those estimates are justified above $q^{2}=40 \mathrm{GeV}^{2}$ (symmetric deviations). Below $40 \mathrm{GeV}^{2}$, the upper limit dominates over the lower limit (unsymmetrical error bars).

\begin{tabular}{cc}
\hline \hline$q^{2}$ & $\left|G\left(q^{2}\right)\right|\left(10^{-3}\right)$ \\
\hline 20 & $4.02(3.51)$ \\
25 & $2.75(1.91)$ \\
30 & $1.97(1.14)$ \\
35 & $1.47(0.73)$ \\
40 & $1.14(0.50)$ \\
45 & $0.900(0.350)$ \\
50 & $0.728(0.255)$ \\
55 & $0.600(0.191)$ \\
60 & $0.502(0.147)$ \\
65 & $0.426(0.115)$ \\
70 & $0.365(0.091)$ \\
75 & $0.316(0.074)$ \\
80 & $0.276(0.060)$ \\
\hline \hline
\end{tabular}

which can be measured presently in several laboratories, for several hyperons, including the $\Omega^{-}[6,12,59-62]$.

Our estimates are presented in Table V. These predictions can be compared with future measurements of the effective form factor $\left|G\left(q^{2}\right)\right|$ of the $\Omega^{-}$baryon.

\section{CONCLUSIONS}

In the last few years, there have been important developments in the experimental study of the electromagnetic structure of baryons in the timelike region, based on $e^{+} e^{-} \rightarrow B \bar{B}$ reactions, for several baryon $(B)$ states. Among other observables, one has access to the effective electromagnetic form factor $\left|G\left(q^{2}\right)\right|$ of the $\Omega^{-}$baryon in the region $\left(q^{2} \geq 4 M_{\Omega}^{2}\right)$.

The recent measurements of the $\Omega^{-}$timelike effective form factor are very pertinent because there is no experimental information about the $\Omega^{-}$electromagnetic structure, apart from the charge and the magnetic moment. There are, however, lattice QCD simulations, at the strange quark physical mass, which can be interpreted as a reliable representation of the physical $\Omega^{-}$(reduced light quark effects). Those lattice QCD simulations are performed in the spacelike region $\left(Q^{2}=-q^{2} \geq 0\right)$ for the electric charge $\left(G_{E 0}\right)$, magnetic dipole $\left(G_{M 1}\right)$, and electric quadrupole $\left(G_{E 2}\right)$ form factors. For the magnetic octupole $\left(G_{M 3}\right)$ form factor, there are only simulations with large errors.

In the present work, we propose using the available information about the $\Omega^{-}$to obtain a complete picture of the electromagnetic structure of the $\Omega^{-}$, in the spacelike region $\left(q^{2} \leq 0\right)$, as well as in the timelike region $\left(q^{2} \geq 4 M_{\Omega}^{2}\right)$. We combine the experimental information (magnetic moment, $q^{2}=0$ ), with the lattice QCD data in 
the spacelike region, and the effective form factor $\left|G\left(q^{2}\right)\right|$ in the timelike region.

We also take into account constraints from pQCD, requiring that $G_{E 0}, G_{M 1} \propto 1 / Q^{4}$ and $G_{E 2}, G_{M 3} \propto 1 / Q^{6}$ for very large $Q^{2}$ (apart logarithmic corrections). In addition, we consider also a condition which relates the $G_{M 1}$ and $G_{M 3}$ form factors in the asymptotic region. The relation $G_{M 1}=\frac{4}{5} \tau G_{M 3}$ has a strong impact on the dependence of the form factors on $Q^{2}$ for very large $Q^{2}$. The discussion of the relevance of the correlation between the from factors $G_{M 1}$ and $G_{M 3}$ is an important contribution of the present work. As far was we know, this relation has not been discussed in the literature. In the present work, the correlation between the magnetic form factors is manifest for a scale of $Q^{2}$ around $900 \mathrm{GeV}^{2}$.

Our analysis is based on the covariant spectator quark model formalism, where the $\Omega^{-}$is a combination of an $S$ state and two $D$ states. The $D$ states are responsible by the deformation of the $\Omega^{-}$system (nonzero results for $G_{E 2}$ and $G_{M 3}$ ). Part of the model (strange quark form factors) was calibrated by previous studies of the decuplet baryon. The parameters related to the $S$ - and $D$-state radial wave functions and the admixture parameters are calibrated by the available physical and lattice QCD data for the $\Omega^{-}$. For the calculations in the timelike region, we use asymptotic relations valid for very large $\left|Q^{2}\right|$. The existing data are described by a small $(0.1 \%) D$-state contribution associated with total quark spin $3 / 2$, and a more significant $(7.6 \%) D$-state contribution associated with total quark spin $1 / 2$. The first contribution dominates $G_{E 2}$, the second contribution dominates $G_{M 3}$. Our results suggest a distribution of charge and magnetism compressed along the spin axis, corresponding to an oblate shape of the $\Omega^{-}$baryon.

We conclude that the available information on the $\Omega^{-}$ baryon, including the lattice QCD data in the spacelike region and the $\left|G\left(q^{2}\right)\right|$ physical data in the timelike region, is compatible with a large value for $G_{M 3}$ near $Q^{2}=0$ $\left[G_{M 3}(0) \simeq 28\right]$ and a fast falloff of $G_{M 3}$ for large $\left|Q^{2}\right|$. It is worth noticing, however, that the present estimations are based on lattice QCD simulations, limited in the range of $Q^{2}$ and precision in the spacelike region, on two timelike data points, and a single lattice calculation of $G_{M 3}$ with a large uncertainty. More timelike data at larger $q^{2}$ are necessary to better constrain the shape of the form factors at large $\left|Q^{2}\right|$ and to increase the impact of the timelike data in the model calibrations.

The value of $G_{M 3}(0)$ is, presently, an open question. The available lattice QCD data are compatible with positive and negative values. Our estimate suggests that $G_{M 3}(0)$ is large and positive, consistent with estimates based on $S U$ (3) quark models and QCD sum rules. Some frameworks, however, point to a small $G_{M 3}$. Accurate simulations possible with the present state-of-the-art lattice QCD techniques are necessary to better infer the magnitude of
$G_{M 3}(0)$ and the shape of the function $G_{M 3}$ at low and intermediate $Q^{2}$.

Future accurate lattice QCD simulations of the four $\Omega^{-}$ form factors at low and intermediate $Q^{2}$ and $e^{+} e^{-} \rightarrow$ $\Omega^{-} \bar{\Omega}^{+}$collision experiments for large values of $q^{2}$ (say $q^{2}>30 \mathrm{GeV}^{2}$ ) are very important to determine the shape of the $\Omega^{-}$form factors and test the present predictions. Those results can also confirm if the model calibrations derived from low- $Q^{2}$ lattice QCD data are also valid in the large- $Q^{2}$ region.

\section{ACKNOWLEDGMENTS}

G. R. was supported by the Fundação de Amparo à Pesquisa do Estado de São Paulo (FAPESP), Project No. 2017/02684-5, Grant No. 2017/17020-BCO-JP.

\section{APPENDIX A: PERTURBATIVE QCD ESTIMATES FOR THE $\Omega^{-}$FORM FACTORS}

We discuss here the asymptotic expressions associated with the form factors $G_{E 0}, G_{M 1}, G_{E 2}$, and $G_{M 3}$. The derivation of those expressions follows the analysis of C. Carlson et al. [94] of the helicity transition amplitudes for very large $Q^{2}$, extended here to $\frac{3}{2}+$ baryons.

\section{Electromagnetic current and form factors}

The current $J^{\mu}$ associated with the $\gamma^{*} \Omega^{-} \bar{\Omega}^{+}$vertex can be written in the Lorentz-covariant and gauge-invariance form $[17,19,21]$

$$
\begin{aligned}
J^{\mu}= & -\left[F_{1}^{*}\left(Q^{2}\right) g^{\alpha \beta}+F_{3}^{*}\left(Q^{2}\right) \frac{q^{\alpha} q^{\beta}}{4 M^{2}}\right] \gamma^{\mu} \\
& -\left[F_{2}^{*}\left(Q^{2}\right) g^{\alpha \beta}+F_{4}^{*}\left(Q^{2}\right) \frac{q^{\alpha} q^{\beta}}{4 M^{2}}\right] \frac{i \sigma^{\mu \nu}}{2 M},
\end{aligned}
$$

in elementary charge units (e). The value of the charge $\left(e_{\Omega}=-1\right)$ is included on the function $F_{1}^{*}\left[F_{1}^{*}(0)=G_{E 0}(0)=-1\right]$.

In the previous expressions, $q$ is the photon momentum, $M$ is the baryon mass, and $F_{i}^{*}(i=1, \ldots, 4)$ are structure form factors. In the following, we use elementary form factors to identify $F_{i}^{*}$. In Eq. (A1), the free indices $(\alpha, \beta)$ are contracted with Rarita-Schwinger spinors $\bar{u}_{\alpha}\left(P_{+}, S_{z}^{\prime}\right)$ and $u_{\beta}\left(P_{-}, S_{z}\right)$ associated with the final and initial states (spin projections $S_{z}^{\prime}$ and $S_{z}$ ), respectively.

The multipole form factors, $G_{E 0}, G_{M 1}, G_{E 2}$, and $G_{M 3}$ can be expressed as linear combinations of the elementary form factors. For the following discussion, however, it is more convenient to define the auxiliary form factors $G_{i}$ $(i=1, \ldots, 4)$,

$$
\begin{aligned}
& G_{1}=F_{1}^{*}-\tau F_{2}^{*}, \\
& G_{2}=F_{1}^{*}+F_{2}^{*},
\end{aligned}
$$




$$
\begin{gathered}
G_{3}=F_{3}^{*}-\tau F_{4}^{*}, \\
G_{4}=F_{3}^{*}+F_{4}^{*} .
\end{gathered}
$$

Using the new notation, we can write [19]

$$
\begin{gathered}
G_{E 0}=\left(1+\frac{2}{3} \tau\right) G_{1}-\frac{1}{3} \tau(1+\tau) G_{3}, \\
G_{M 1}=\left(1+\frac{4}{5} \tau\right) G_{2}-\frac{2}{5} \tau(1+\tau) G_{4}, \\
G_{E 2}=G_{1}-\frac{1}{2}(1+\tau) G_{3}, \\
G_{M 3}=G_{2}-\frac{1}{2}(1+\tau) G_{4} .
\end{gathered}
$$

The inverse relations are

$$
\begin{aligned}
& F_{1}^{*}=\frac{1}{1+\tau}\left(G_{1}+\tau G_{2}\right), \\
& F_{2}^{*}=-\frac{1}{1+\tau}\left(G_{1}-G_{2}\right), \\
& F_{3}^{*}=\frac{2}{1+\tau}\left(G_{3}+\tau G_{4}\right), \\
& F_{4}^{*}=-\frac{2}{1+\tau}\left(G_{3}-G_{4}\right),
\end{aligned}
$$

and

$$
\begin{gathered}
G_{1}=G_{E 0}-\frac{2}{3} \tau G_{E 2}, \\
G_{2}=G_{M 1}-\frac{4}{5} \tau G_{M 3}, \\
G_{3}=\frac{2}{1+\tau} G_{E 0}-\frac{2}{1+\tau}\left(1+\frac{2}{3} \tau\right) G_{E 2}, \\
G_{4}=\frac{2}{1+\tau} G_{M 1}-\frac{2}{1+\tau}\left(1+\frac{4}{5} \tau\right) G_{M 3} .
\end{gathered}
$$

\section{Breit frame transition amplitudes}

The study of the asymptotic behavior of the electromagnetic form factors is simplified when we calculate the transition amplitudes between the possible spin projections in a given frame. Following C. Carlson, we consider the amplitudes in the Breit frame [94]

$$
\begin{aligned}
G_{+} & =\left\langle J^{+}\right\rangle_{+\frac{1}{2},-\frac{1}{2}}, \\
G_{01} & =\left\langle J^{0}\right\rangle_{+\frac{1}{2},+\frac{1}{2}},
\end{aligned}
$$

$$
\begin{aligned}
& G_{03}=\left\langle J^{0}\right\rangle_{+\frac{3}{2},+\frac{3}{2}}, \\
& G_{-}=\left\langle J^{+}\right\rangle_{+\frac{3}{2},+\frac{1}{2}},
\end{aligned}
$$

where

$$
\begin{aligned}
\left\langle J^{0}\right\rangle_{S_{z}^{\prime}, S_{z}} & =\left\langle+\frac{1}{2} \mathbf{q}, S_{z}^{\prime}\left|J^{0}\right|-\frac{1}{2} \mathbf{q}, S_{z}\right\rangle, \\
\left\langle J^{+}\right\rangle_{S_{z}^{\prime}, S_{z}} & =\left\langle+\frac{1}{2} \mathbf{q}, S_{z}^{\prime}\left|J^{+}\right|-\frac{1}{2} \mathbf{q}, S_{z}\right\rangle,
\end{aligned}
$$

and $J^{+}=-\frac{1}{\sqrt{2}}\left(J^{1}+i J^{2}\right)$. Note that in the Breit frame the initial state has helicity $-2 S_{z}$ and the final-state helicity $2 S_{z}^{\prime}$.

The notation $G_{\ell}(\ell=+, 01,03,-)$ is inspired on the notation from Refs. [94] for the transitions between spin$1 / 2$ states to spin- $1 / 2$ or spin-3/2 states (like the $\gamma^{*} N \rightarrow \Delta$ transition), where $\ell= \pm, 0$ are related to the photon polarization vectors. In the present case, however, it is necessary to distinguish between two scalar transitions: $S_{z}=+\frac{1}{2} \rightarrow S_{z}^{\prime}=+\frac{1}{2}$, labeled as $G_{01}$, and $S_{z}=+\frac{3}{2} \rightarrow$ $S_{z}^{\prime}=+\frac{3}{2}$, labeled as $G_{03}$. The amplitude $G_{03}$ is exclusive of the spin-3/2 elastic transitions.

The Eqs. (A18)-(A21) represent the four independent, nonzero amplitudes. The omitted cases can be related to these four cases.

The explicit calculation of the amplitudes (A18)-(A21) gives [19]

$$
\begin{gathered}
G_{+}=-\frac{2 \sqrt{2}}{3} \sqrt{\tau}\left[G_{M 1}+\frac{6}{5} \tau G_{M 3}\right], \\
G_{01}=G_{1}+\frac{4}{3} \tau G_{E 2}=G_{E 0}+\frac{2}{3} \tau G_{E 2}, \\
G_{03}=G_{1}=G_{E 0}-\frac{2}{3} \tau G_{E 2}, \\
G_{-}=-\sqrt{\frac{2}{3}} \sqrt{\tau} G_{2} .
\end{gathered}
$$

In the calculations, we use the normalization $\bar{u}_{\alpha}\left(P, S_{z}^{\prime}\right) u^{\alpha}\left(P, S_{z}\right)=-\delta_{S_{z}^{\prime} S_{z}}$ in the limit $P=(M, \mathbf{0})$.

\section{Large- $Q^{2}$ behavior}

One can now use the pQCD analysis from C. Carlson et al. $[94,95]$. In the leading-order amplitude $\left(G_{+}\right)$, the electromagnetic interaction preserves the helicity of the initial state (helicity conservation), meaning that there is no spin flip of any quark. In the remaining amplitudes, the helicity is modified by the spin flip of one or more quarks. Each spin flip transition is suppressed by a factor $m_{q} / Q$, where $m_{q}$ is the current quark mass [94]. The consequence of this suppression is that $G_{+}$has the slowest falloff with 
$Q^{2}$ for large $Q^{2}$, and the remaining amplitudes are further suppressed by multiple factors $m_{q} / Q$.

The asymptotic behavior of $G_{\ell}$ can be ordered as

$$
\begin{aligned}
G_{+} & \propto \frac{1}{Q^{3}}, \\
G_{01} & \propto \frac{1}{Q^{4}}, \\
G_{03} & \propto \frac{1}{Q^{4}}, \\
G_{-} & \propto \frac{1}{Q^{5}} .
\end{aligned}
$$

The amplitudes $G_{01}$ and $G_{03}$ have the same behavior because they are associated with the same helicity variation between the initial and the final state.

The $\mathrm{pQCD}$ constraints on the transition form factors can now be derived based on the comparison of the general relations (A24)-(A27) and the asymptotic expressions (A28)-(A31). We start our analysis with the relation for $G_{-}$. From the comparison between (A27) with the asymptotic result (A31), we conclude that

$$
G_{2}=G_{M 1}-\frac{4}{5} \tau G_{M 3}=\mathcal{O}\left(\frac{1}{Q^{6}}\right) .
$$

From the relations for $G_{01}$ and $G_{03}$, one can conclude from the comparison of the Eqs. (A25) and (A26) with the relations (A29) and (A30), respectively, that

$$
G_{E 0} \pm \frac{2}{3} \tau G_{E 2}=\mathcal{O}\left(\frac{1}{Q^{4}}\right)
$$

meaning that

$$
G_{E 0}, \tau G_{E 2} \propto \frac{1}{Q^{4}} .
$$

To finish the analysis, we look for the amplitude $G_{+}$. Using Eqs. (A24), (A28), and (A32), one obtains

$$
G_{+}=-\frac{5 \sqrt{2}}{3} \sqrt{\tau}\left[G_{M 1}+\mathcal{O}\left(\frac{1}{Q^{6}}\right)\right] \propto \frac{1}{Q^{3}},
$$

from which we conclude that

$$
G_{M 1} \propto \frac{1}{Q^{4}} .
$$

Combining this result with (A32), we conclude also that

$$
\tau G_{M 3} \propto \frac{1}{Q^{4}} .
$$

A corollary of the relations (A34), (A36), and (A37) is that $G_{E 0} \propto \tau G_{E 2} \propto 1 / Q^{4}$ and $G_{M 1} \propto \tau G_{M 3} \propto 1 / Q^{4}$.

\section{Summary of the pQCD limit}

We can now summarize the expected results for the falloff of the multipole form factors at very large $Q^{2}$.

For very large $Q^{2}$ (pQCD regime), the transition amplitudes $G_{\ell}$ follow the power law falloffs from (A28)-(A31). The first consequence of the general analysis is that

$$
\begin{aligned}
& G_{E 0}, \quad G_{M 1} \propto \frac{1}{Q^{4}}, \\
& G_{E 2}, \quad G_{M 3} \propto \frac{1}{Q^{6}} .
\end{aligned}
$$

The analysis of the amplitude $G_{-}$, determined by $G_{2}$, demonstrates that, in addition to (A38) and (A39), for $\tau \gg 1$, one has also

$$
G_{M 1} \simeq \frac{4}{5} \tau G_{M 3} .
$$

The implication of the previous relation is that the two magnetic form factors are correlated for very large $Q^{2}$.

The relations $G_{M 1} \propto 1 / Q^{4}, G_{M 3} \propto 1 / Q^{6}$ and (A40) imply that the leading-order the terms in $G_{M 1}$ and $\tau G_{M 3}$ are given by terms of order $1 / Q^{4}$, which cancel exactly in $G_{M 1}-\frac{4}{5} \tau G_{M 3}$, and only the terms of higher order $1 / Q^{6}$ survive.

To the best of our knowledge, the relation (A40), valid for very large $Q^{2}$, has not been discussed in the literature. We emphasize, however, that the relations (A38), (A39), and (A40) are the consequence of the natural order of the amplitudes (A18)-(A21).

The leading-order falloff of the form factors $F_{i}^{*}$ and $G_{i}$ $(i=1, \ldots, 4)$ can also be estimated, based on the relations between representations (A2)-(A5), (A6)-(A9), (A10)(A13), and (A14)-(A17). Those results are presented in the first row (case 1) of Table VI.

\section{Alternative forms for the form factors falloffs}

The results (A38), (A39), and (A40) are then a consequence of the $\mathrm{pQCD}$ regime. One can, nevertheless, look for weaker constraints at large $Q^{2}$.

If we remove the condition (A40) but keep the conditions (A38) and (A39), we obtain different falloffs for the form factors $F_{i}^{*}$ and $G_{i}(i=1, \ldots, 4)$. The corresponding forms are presented in the second row (case 2) of Table VI.

TABLE VI. Expected falloffs according with different large- $Q^{2}$ conditions. Case 1: consequence of the conditions (A38), (A39), and (A40). Case 2: ignore the condition (A40).

\begin{tabular}{l|cccc|cccc}
\hline \hline & $G_{1}$ & $G_{2}$ & $G_{3}$ & $G_{4}$ & $F_{1}^{*}$ & $F_{2}^{*}$ & $F_{3}^{*}$ & $F_{4}^{*}$ \\
\hline Case 1 & $\frac{1}{Q^{4}}$ & $\frac{1}{Q^{6}}$ & $\frac{1}{Q^{6}}$ & $\frac{1}{Q^{8}}$ & $\frac{1}{Q^{6}}$ & $\frac{1}{Q^{6}}$ & $\frac{1}{Q^{8}}$ & $\frac{1}{Q^{8}}$ \\
Case 2 & $\frac{1}{Q^{4}}$ & $\frac{1}{Q^{4}}$ & $\frac{1}{Q^{6}}$ & $\frac{1}{Q^{6}}$ & $\frac{1}{Q^{4}}$ & $\frac{1}{Q^{6}}$ & $\frac{1}{Q^{6}}$ & $\frac{1}{Q^{8}}$ \\
\hline \hline
\end{tabular}


The main consequence in the model which ignores the condition (A40) appears in the amplitude $G_{-}$. As before, we obtain $G_{+} \propto 1 / Q^{3}, G_{01}$, and $G_{03} \propto 1 / Q^{4}$. In the case of $G_{-}$, since $G_{-} \propto Q G_{2}$ and $G_{2} \propto 1 / Q^{4}\left(\right.$ instead of $\left.G_{2} \propto 1 / Q^{6}\right)$, one obtains

$$
G_{-} \propto \frac{1}{Q^{3}},
$$

in contradiction with the asymptotic relation (A31) expected from $\mathrm{pQCD}$.

In summary, if we ignore the relation (A32), we fail to reproduce the natural order of the amplitudes: $G_{+} \propto 1 / Q^{3}$, $G_{01}, G_{03} \propto 1 / Q^{4}$, and $G_{-} \propto 1 / Q^{5}$.

\section{APPENDIX B: ASYMPTOTIC RELATIONS FOR THE COVARIANT SPECTATOR QUARK MODEL}

We discuss in this Appendix the asymptotic relations associated with the electromagnetic form factors of the $\Omega^{-}$ from Eqs. (4.12)-(4.15), derived from the covariant spectator quark model framework.

\section{Uncorrelated form factors}

In a first step, we ignore the constraint (2.8). From the discussion from Sec. IV D, we know already that

$$
\mathcal{I}_{S} \propto \frac{1}{Q^{4}}, \quad \mathcal{I}_{D 3} \propto \frac{1}{Q^{4}}, \quad \mathcal{I}_{D 1} \propto \frac{1}{Q^{4}},
$$

apart logarithmic corrections.

For the discussion of the large- $Q^{2}$ region, it is important to notice that the functions $\tilde{g}_{\Omega}$ and $\tilde{f}_{\Omega}$, related with the strange quark form factors $f_{10}\left(Q^{2}\right)$ and $f_{20}\left(Q^{2}\right)$, behave as constants for very large $Q^{2}$,

$$
\begin{gathered}
\tilde{g}_{\Omega} \rightarrow \kappa_{q}^{\prime}-\lambda_{q}, \\
\tilde{f}_{\Omega} \rightarrow-\lambda_{q},
\end{gathered}
$$

where $\kappa_{q}^{\prime}=\kappa_{s}\left[d_{0} \frac{m_{\phi}^{2}}{4 M_{N} M_{\Omega}}+\left(1-d_{0}\right) \frac{M_{h}^{2}}{4 M_{N} M_{\Omega}}\right]$. All parameters are defined in the strange quark form factors. From our calibration to the strange quark current [4], one has $\lambda_{q}=$ 1.21 and $\kappa_{q}^{\prime}=1.84$.
From Eqs. (B2) and (B3), one can conclude that for large $Q^{2}, \tilde{f}_{\Omega}$ is negative, and $\tilde{g}_{\Omega}$ is positive. At low $Q^{2}$, these functions are both negative.

The combination of the results (B1) with Eqs. (4.12)(4.15) leads to the $G_{E 0}, G_{M 1} \propto 1 / Q^{4}$ and $G_{E 2}, G_{M 3} \propto 1 / Q^{6}$.

We now look for the result for $G_{M 1}-\frac{4}{5} \tau G_{M 3}$. From Eqs. (4.13) and (4.15), one obtains

$$
G_{M 1}-\frac{4}{5} \tau G_{M 3}=N^{2} \tilde{f}_{\Omega}\left[\mathcal{I}_{S}-2 b \mathcal{I}_{D 1}\right]
$$

From this result, we conclude that the relation between the two magnetic form factors is independent from the $D 3$ component.

If the functions $\mathcal{I}_{S}$ and $b \mathcal{I}_{D 1}$ are uncorrelated, we can conclude that $G_{M 1}-\frac{4}{5} \tau G_{M 3} \propto 1 / Q^{4}$. Working backward, we can derive the corresponding falloff for the form factors $G_{i}$ and $F_{i}^{*}(i=1,2,3,4)$ and obtain the relations associated with the case 2 from Table VI, discussed in Appendix A.

\section{Asymptotic relations consistent with $\mathbf{p Q C D}$}

We consider now the possibility of imposing that the results of the covariant spectator quark model are consistent with the relation (2.8) for very large $Q^{2}$. From (B4), we conclude that the condition (2.8) is valid if

$$
\mathcal{I}_{S}=2 b \mathcal{I}_{D 1}
$$

apart terms of the order of $1 / Q^{6}$, since it was established already that the overlap integrals are of the order of $1 / Q^{4}$.

The compatibility of the covariant spectator quark model with $\mathrm{PQCD}$ requires then that $\mathcal{I}_{S}=b \mathcal{I}_{D 1}$. The connection with between $b \mathcal{I}_{D 1}$ and $\mathcal{I}_{S}$ can be imposed numerically.

Combining the original expressions (4.12)-(4.15) for the form factors with the condition (B5), we can write for large $Q^{2}$

$$
\begin{gathered}
G_{E 0}=N^{2} \tilde{g}_{\Omega} \mathcal{I}_{S}, \\
\tau G_{E 2}=N^{2} \tilde{g}_{\Omega} a \mathcal{I}_{D 3}, \\
G_{M 1}=\frac{4}{5} N^{2} \tilde{f}_{\Omega}\left[\mathcal{I}_{S}+a \mathcal{I}_{D 3}\right], \\
\tau G_{M 3}=N^{2} \tilde{f}_{\Omega}\left[\mathcal{I}_{S}+a \mathcal{I}_{D 3}\right] .
\end{gathered}
$$

[1] M. A. B. Beg, B. W. Lee, and A. Pais, Phys. Rev. Lett. 13, 514 (1964).

[2] S. Capstick and W. Roberts, Prog. Part. Nucl. Phys. 45, S241 (2000).
[3] G. Muller, Quantum Mechanics Symmetries, (SpringVerlag, Berlin, 1994).

[4] G. Ramalho, K. Tsushima, and F. Gross, Phys. Rev. D 80, 033004 (2009). 
[5] G. Ramalho and M. T. Peña, Phys. Rev. D 83, 054011 (2011).

[6] G. Ramalho, M. T. Peña, and K. Tsushima, Phys. Rev. D 101, 014014 (2020).

[7] C. Alexandrou, T. Korzec, G. Koutsou, J. W. Negele, and Y. Proestos, Phys. Rev. D 82, 034504 (2010).

[8] H. T. Diehl et al., Phys. Rev. Lett. 67, 804 (1991).

[9] N. B. Wallace, P. M. Border, D. P. Ciampa, G. Guglielmo, K. J. Heller, D. M. Woods, K. A. Johns, Y. T. Gao, M. J. Longo, and R. Rameika, Phys. Rev. Lett. 74, 3732 (1995).

[10] P. A. Zyla et al. (Particle Data Group), Prog. Theor. Exp. Phys. 2020, 083C01 (2020).

[11] A. Afanasev et al., JLAB-PR-12-008, https://inspirehep .net/literature/1210267.

[12] S. Dobbs, A. Tomaradze, T. Xiao, K. K. Seth, and G. Bonvicini, Phys. Lett. B 739, 90 (2014).

[13] S. Boinepalli, D. B. Leinweber, P. J. Moran, A. G. Williams, J. M. Zanotti, and J. B. Zhang, Phys. Rev. D 80, 054505 (2009).

[14] I. G. Aznauryan et al., Int. J. Mod. Phys. E 22, 1330015 (2013).

[15] G. Ramalho, Few Body Syst. 59, 92 (2018).

[16] I. G. Aznauryan and V. D. Burkert, Prog. Part. Nucl. Phys. 67, 1 (2012).

[17] S. Nozawa and D. B. Leinweber, Phys. Rev. D 42, 3567 (1990).

[18] V. Pascalutsa, M. Vanderhaeghen, and S. N. Yang, Phys. Rep. 437, 125 (2007).

[19] C. Alexandrou, T. Korzec, G. Koutsou, C. Lorce, J. W. Negele, V. Pascalutsa, A. Tsapalis, and M. Vanderhaeghen, Nucl. Phys. A825, 115 (2009).

[20] G. Ramalho and M. T. Peña, J. Phys. G 36, 085004 (2009).

[21] G. Ramalho, M. T. Peña, and F. Gross, Phys. Rev. D 81, 113011 (2010); Phys. Lett. B 678, 355 (2009).

[22] G. Ramalho, M. T. Peña, and A. Stadler, Phys. Rev. D 86, 093022 (2012).

[23] C. W. Bernard, T. Draper, K. Olynyk, and M. Rushton, Phys. Rev. Lett. 49, 1076 (1982).

[24] Y. Tomozawa, Phys. Rev. D 25, 795 (1982).

[25] H. Georgi and A. Manohar, Phys. Lett. 132B, 183 (1983).

[26] M. I. Krivoruchenko, Yad. Fiz. 45, 169 (1987) [Sov. J. Nucl. Phys. 45, 109 (1987)], https://old.inspirehep.net/record/ 209822.

[27] J. H. Kim, C. H. Lee, and H. K. Lee, Nucl. Phys. A501, 835 (1989).

[28] J. Kunz and P. J. Mulders, Phys. Rev. D 41, 1578 (1990).

[29] K. T. Chao, Phys. Rev. D 41, 920 (1990).

[30] C. Gobbi, S. Boffi, and D. O. Riska, Nucl. Phys. A547, 633 (1992).

[31] B. Schwesinger and H. Weigel, Nucl. Phys. A540, 461 (1992).

[32] F. Schlumpf, Phys. Rev. D 48, 4478 (1993).

[33] S. T. Hong and G. E. Brown, Nucl. Phys. A580, 408 (1994).

[34] M. N. Butler, M. J. Savage, and R. P. Springer, Phys. Rev. D 49, 3459 (1994).

[35] P. Ha, Phys. Rev. D 58, 113003 (1998).

[36] J. Linde, T. Ohlsson, and H. Snellman, Phys. Rev. D 57, 5916 (1998).

[37] S. L. Zhu, W. Y. P. Hwang, and Z. S. P. Yang, Phys. Rev. D 57, 1527 (1998).
[38] G. Wagner, A. J. Buchmann, and A. Faessler, J. Phys. G 26, 267 (2000).

[39] T. M. Aliev, A. Ozpineci, and M. Savci, Phys. Rev. D 62 , 053012 (2000).

[40] A. Iqubal, M. Dey, and J. Dey, Phys. Lett. B 477, 125 (2000).

[41] B. O. Kerbikov and Yu. A. Simonov, Phys. Rev. D 62, 093016 (2000).

[42] J. Franklin, Phys. Rev. D 66, 033010 (2002).

[43] C. S. An, Q. B. Li, D. O. Riska, and B. S. Zou, Phys. Rev. C 74, 055205 (2006); 75, 069901(E) (2007).

[44] S. S. Gershtein and Yu. M. Zinovev, Yad. Fiz. 33, 1442 (1981) [Sov. J. Nucl. Phys. 33, 772 (1981)], https://old .inspirehep.net/record/164467.

[45] J. M. Richard, Z. Phys. C 12, 369 (1982).

[46] N. Isgur, G. Karl, and R. Koniuk, Phys. Rev. D 25, 2394 (1982).

[47] M. I. Krivoruchenko and M. M. Giannini, Phys. Rev. D 43, 3763 (1991).

[48] D. B. Leinweber, T. Draper, and R. M. Woloshyn, Phys. Rev. D 46, 3067 (1992).

[49] A. J. Buchmann and E. M. Henley, Phys. Rev. D 65, 073017 (2002); A. J. Buchmann and R. F. Lebed, Phys. Rev. D 67, 016002 (2003).

[50] D. Arndt and B. C. Tiburzi, Phys. Rev. D 68, 114503 (2003); 69, 059904(E) (2004).

[51] T. Ledwig, A. Silva, and M. Vanderhaeghen, Phys. Rev. D 79, 094025 (2009).

[52] T. M. Aliev, K. Azizi, and M. Savci, Phys. Lett. B 681, 240 (2009).

[53] L. S. Geng, J. Martin Camalich, and M. J. Vicente Vacas, Phys. Rev. D 80, 034027 (2009).

[54] H. S. Li, Z. W. Liu, X. L. Chen, W. Z. Deng, and S. L. Zhu, Phys. Rev. D 95, 076001 (2017).

[55] J. Y. Kim and H. C. Kim, Eur. Phys. J. C 79, 570 (2019).

[56] A. J. Buchmann and E. M. Henley, Eur. Phys. J. A 35, 267 (2008).

[57] D. Nicmorus, G. Eichmann, and R. Alkofer, Phys. Rev. D 82, 114017 (2010).

[58] H. Sanchis-Alepuz, R. Williams, and R. Alkofer, Phys. Rev. D 87, 096015 (2013).

[59] B. Aubert et al. (BABAR Collaboration), Phys. Rev. D 73, 012005 (2006); 76, 092006 (2007).

[60] M. Ablikim et al. (BESIII Collaboration), Phys. Rev. D 87, 112011 (2013); 97, 032013 (2018); M. Ablikim et al. (BESIII Collaboration), Phys. Lett. B 814, 136110 (2021).

[61] S. Dobbs, K. K. Seth, A. Tomaradze, T. Xiao, and G. Bonvicini, Phys. Rev. D 96, 092004 (2017).

[62] B. Singh et al. (PANDA Collaboration), Phys. Rev. D 95, 032003 (2017).

[63] N. Cabibbo and R. Gatto, Phys. Rev. 124, 1577 (1961).

[64] P. Kroll, T. Pilsner, M. Schurmann, and W. Schweiger, Phys. Lett. B 316, 546 (1993).

[65] R. Jakob, P. Kroll, M. Schurmann, and W. Schweiger, Z. Phys. A 347, 109 (1993).

[66] R. L. Jaffe and F. Wilczek, Phys. Rev. Lett. 91, 232003 (2003).

[67] F. Wilczek, in From Fields to Strings: Circumnavigating Theoretical Physics, edited by M. Shifman et al. (World 
Scientific, Singapore, 2005), Vol. 1, pp. 77-93, 10.1142/ 9789812775344_0007.

[68] A. Selem and F. Wilczek, New Trends HERA Phys. 2005, 337 (2006).

[69] J. G. Korner and M. Kuroda, Phys. Rev. D 16, 2165 (1977).

[70] A. Z. Dubnickova, S. Dubnicka, and P. Strizenec, Czech. J. Phys. 43, 1177 (1993).

[71] L. L. Liu, C. Wang, Y. Liu, and X. H. Guo, Phys. Rev. D 95, 054001 (2017).

[72] E. Perotti, G. Fäldt, A. Kupsc, S. Leupold, and J. J. Song, Phys. Rev. D 99, 056008 (2019).

[73] J. Haidenbauer, T. Hippchen, K. Holinde, B. Holzenkamp, V. Mull, and J. Speth, Phys. Rev. C 45, 931 (1992).

[74] J. Haidenbauer, U. G. Meißner, and L. Y. Dai, Phys. Rev. D 103, 014028 (2021).

[75] O. D. Dalkarov, P. A. Khakhulin, and A. Y. Voronin, Nucl. Phys. A833, 104 (2010).

[76] J. Haidenbauer and U. G. Meissner, Phys. Lett. B 761, 456 (2016).

[77] X. Cao, J. P. Dai, and Y. P. Xie, Phys. Rev. D 98, 094006 (2018).

[78] Y. Yang, D. Y. Chen, and Z. Lu, Phys. Rev. D 100, 073007 (2019).

[79] G. Fäldt and A. Kupsc, Phys. Lett. B 772, 16 (2017).

[80] Z. Y. Li and J. J. Xie, arXiv:2012.02379.

[81] F. Gross, G. Ramalho, and M. T. Peña, Phys. Rev. C 77, 015202 (2008).

[82] F. Gross, G. Ramalho, and M. T. Peña, Phys. Rev. D 85, 093005 (2012); F. Gross, G. Ramalho, and M. T. Peña, Phys. Rev. D 85, 093006 (2012).

[83] G. Ramalho, M. T. Peña, and F. Gross, Eur. Phys. J. A 36, 329 (2008).

[84] G. Ramalho, M. T. Peña, and F. Gross, Phys. Rev. D 78, 114017 (2008).

[85] A. J. Buchmann and E. M. Henley, Phys. Rev. C 63, 015202 (2000).

[86] C. Aubin, K. Orginos, V. Pascalutsa, and M. Vanderhaeghen, Phys. Rev. D 79, 051502(R) (2009).

[87] E. E. Jenkins and A. V. Manohar, Phys. Lett. B 255, 558 (1991).

[88] U. G. Meissner and S. Steininger, Nucl. Phys. B499, 349 (1997).

[89] V. Bernard, Prog. Part. Nucl. Phys. 60, 82 (2008).

[90] S. Pacetti, R. B. Ferroli, and E. Tomasi-Gustafsson, Phys. Rep. 550-551, 1 (2015).

[91] C. Tzara, Nucl. Phys. B18, 246 (1970).

[92] A. Denig and G. Salme, Prog. Part. Nucl. Phys. 68, 113 (2013).

[93] J. Haidenbauer, X.-W. Kang, and U.-G. Meissner, Nucl. Phys. A929, 102 (2014).

[94] C. E. Carlson and N. C. Mukhopadhyay, Phys. Rev. Lett. 81, 2646 (1998); C. E. Carlson, Phys. Rev. D 34, 2704 (1986).

[95] C. E. Carlson, Few Body Syst. Suppl. 11, 10 (1999).

[96] G. P. Lepage and S. J. Brodsky, Phys. Rev. D 22, 2157 (1980); S. J. Brodsky and G. R. Farrar, Phys. Rev. D 11, 1309 (1975); S. J. Brodsky and G. R. Farrar, Phys. Rev. Lett. 31, 1153 (1973).

[97] R. C. E. Devenish, T.S. Eisenschitz, and J. G. Korner, Phys. Rev. D 14, 3063 (1976).
[98] G. Ramalho and M. T. Peña, Phys. Rev. D 89, 094016 (2014).

[99] G. Eichmann and G. Ramalho, Phys. Rev. D 98, 093007 (2018).

[100] G. Ramalho, Phys. Rev. D 93, 113012 (2016).

[101] F. Gross, Phys. Rev. 186, 1448 (1969); F. Gross, J. W. Van Orden, and K. Holinde, Phys. Rev. C 45, 2094 (1992).

[102] G. Ramalho and M. T. Peña, Phys. Rev. D 80, 013008 (2009).

[103] F. Gross, G. Ramalho, and K. Tsushima, Phys. Lett. B 690 , 183 (2010); G. Ramalho and K. Tsushima, Phys. Rev. D 87, 093011 (2013).

[104] G. Ramalho, Eur. Phys. J. A 54, 75 (2018); Phys. Rev. D 94, 114001 (2016).

[105] G. Ramalho, Phys. Rev. D 102, 054016 (2020); G. Ramalho and K. Tsushima, Phys. Rev. D 88, 053002 (2013); G. Ramalho and K. Tsushima, Phys. Rev. D 87, 093011 (2013); G. Ramalho and K. Tsushima, Phys. Rev. D 86, 114030 (2012).

[106] G. Ramalho and M. T. Peña, J. Phys. G 36, 115011 (2009).

[107] G. Ramalho and K. Tsushima, Phys. Rev. D 81, 074020 (2010); 89, 073010 (2014); 82, 073007 (2010).

[108] G. Ramalho, Phys. Rev. D 95, 054008 (2017); G. Ramalho, Phys. Rev. D 90, 033010 (2014).

[109] G. Ramalho and M. T. Peña, Phys. Rev. D 84, 033007 (2011); G. Ramalho, D. Jido, and K. Tsushima, Phys. Rev. D 85, 093014 (2012).

[110] G. Ramalho, Phys. Rev. D 102, 054016 (2020).

[111] G. Ramalho, M. T. Peña, J. Weil, H. van Hees, and U. Mosel, Phys. Rev. D 93, 033004 (2016); G. Ramalho and M. T. Peña, Phys. Rev. D 85, 113014 (2012).

[112] G. Ramalho and M. T. Peña, Phys. Rev. D 101, 114008 (2020); 95, 014003 (2017).

[113] G. Ramalho, K. Tsushima, and A. W. Thomas, J. Phys. G 40, 015102 (2013); G. Ramalho, J. P. B. C. de Melo, and K. Tsushima, Phys. Rev. D 100, 014030 (2019).

[114] G. Ramalho and K. Tsushima, Phys. Rev. D 94, 014001 (2016).

[115] F. Gross, G. Ramalho, and M. T. Peña, Phys. Rev. C 77, 035203 (2008).

[116] W. Rarita and J. Schwinger, Phys. Rev. 60, 61 (1941).

[117] M. Benmerrouche, R. M. Davidson, and N. C. Mukhopadhyay, Phys. Rev. C 39, 2339 (1989).

[118] Reference [83] presents the demonstration only for the case $\psi_{S} \propto 1 /(\alpha+\chi)^{2}$, but the methodology can be generalized for $\psi_{S} \propto 1 /\left(\left(\alpha_{1}+\chi\right)\left(\alpha_{2}+\chi\right)\right)$. We obtain the same result in leading order, except that the normalization factor $N_{S}$ is determined by a different condition, which depends on $\alpha_{1}$ and $\alpha_{2}$.

[119] Using the arguments of Appendix G from Ref. [83], we can conclude that the term $1 /\left(\left(\alpha_{1}+\chi\right)\left(\alpha_{2}+\chi\right)\right)$ contributes to a term of the order $1 / Q^{4}$ apart logarithmic corrections, and the term $1 /\left[\left(\alpha_{1}^{\prime}+\chi\right)\left(\alpha_{2}^{\prime}+\chi\right)^{2}\right]$ contributes to a term of the order $1 / Q^{6}$ for very large $Q^{2}$.

[120] O. Gayou et al. (Jefferson Lab Hall A Collaboration), Phys. Rev. Lett. 88, 092301 (2002).

[121] A. J. R. Puckett et al., Phys. Rev. C 96, 055203 (2017); 98, 019907(E) (2018). 